\title{
A diagrammatic description of the equations of motion, current, and noise within the second-order von Neumann approach
}

\author{
O Karlström ${ }^{1}$, C Emary ${ }^{2}$, P Zedler ${ }^{2}$, J N Pedersen P $^{1,3}$, \\ C Bergenfeldt ${ }^{1}$, P Samuelsson ${ }^{1}$, T Brandes ${ }^{2}$ and A Wacker $^{1}$ \\ ${ }^{1}$ Mathematical Physics, University of Lund, Box 118, SE-22100 Lund, Sweden \\ ${ }^{2}$ Institut für Theoretische Physik, Technische Universität Berlin, D-10623 \\ Berlin, Germany \\ ${ }^{3}$ Department of Micro- and Nanotechnology (DTU Nanotech), Technical \\ University of Denmark, Building 345 East, DK-2800 Kongens Lyngby, Denmark.
}

\begin{abstract}
We investigate the second-order von Neumann approach from a diagrammatic point-of-view and demonstrate its equivalence with the resonant tunneling approximation. Investigation of higher-order diagrams shows that the method correctly reproduces the equation of motion for the single-particle reduced density matrix of an arbitrary non-interacting many-body system. This explains why the method reproduces the current exactly for such systems. We go on to show, however, that diagrams not included in the method are needed to calculate exactly higher cumulants of the charge transport. This thorough comparison sheds light on the validity of all these self consistent second-order approaches. We analyze the discrepancy between the noise calculated by our method and the exact Levitov formula for a simple non-interacting quantum dot model. Furthermore we study the noise of the canyon of current suppression in a two-level dot, a phenomenon that requires the inclusion of electron-electron interaction as well as higher-order tunneling processes.
\end{abstract}

PACS numbers: 72.70.+m,73.63.-b,05.60.Gg,73.23.Hk 


\section{INTRODUCTION}

Understanding transport through interacting nanostructures is of major importance for the development of nanoelectronics. The last decades have seen a high activity within the field, but calculating the transport through general interacting systems remains a challenging task. In contrast, transport through non-interacting systems have long-since been well explained by transmission formalism [1]. In this framework the Full Counting Statistics (FCS) was later developed by Levitov, Lee, and Lesovik [2]. Lately it was also shown how the exact equations of motion (EOM) for the reduced density matrix could be derived [3, 4. In a very recent work it was shown how this could be used for deriving the FCS in the noninteracting limit [5]. Although very useful in their regime of validity, these methods are restricted to non-interacting systems, and can generally not be applied to study the transport of confined nanostructures, where electron-electron-interactions are of major importance. The transmission formalism can also be used at equilibrium configurations where the occupation of the dot is well defined. Here, we are interested in nonequilibrium, where the dot is fractionally occupied.

To deal with the complications of interacting systems, various methods have been developed. The most widely used technique is the generalized master equation approach which can be derived in many different ways including the real-time diagrammatic technique [6, 7] by Schoeller, König, Schön, and co-workers, as well as the Bloch-Redfield approach originally developed in Refs. 8, 9, 10, Comparisons of different approaches have been performed in Refs. 11, 12. Bloch-Redfield approaches result in a hierarchy of coupled linear differential equations, which must be truncated. Keeping only the lowest non-vanishing order in dot-lead couplings one arrives at firstorder methods, valid in the regime where sequential tunneling is dominant. Then one neglects the effects of co-tunneling, giving important contributions for stronger couplings [13, 14, 15, 16. Keeping higher-order terms, methods such as the second order von Neumann $(2 \mathrm{vN})$ approach can be derived [17, where co-tunneling as well as the coherence between the dot states are included. Lately Jin et al. developed a method [18] which, at a certain level of approximation was shown to be equivalent to the resonant tunneling approximation within the real-time diagrammatic technique. Equivalence with the $2 \mathrm{vN}$ approach was also suggested.

The aim of this paper is to investigate the $2 \mathrm{vN}$ approach from a diagrammatic point-of-view. This allows us to establish a number of exact results for the theory. Firstly, we demonstrate the equivalence between the $2 \mathrm{vN}$ method and the resonant tunneling approximation, (and thus the method of Jin et al). This proof is enabled by the compact notation of Liouville diagrams. We then consider non-interacting systems and show that, although the $2 \mathrm{vN}$ approach yields the correct EOM for the reduced single-particle density matrix $p$, enabling an exact calculation of the current [18, the EOM for the complete reduced many-body density matrix $\rho$ is not exact. This is shown via the cancelling of higher order diagrams. To see how the cancelling works we have apply Keldysh diagrams. These provide a more detailed description, and the Liouville diagrams are actually sums over distinct Keldysh diagrams. Building on the work of Ref. [19] we consider the $2 \mathrm{vN}$ approach with the inclusion of counting fields. Using our diagrammatic method, we are able to explain why the shot-noise calculated for a non-interacting system is not exact, even though the current is. If fact, we find that the inclusion of diagrams of order $\Gamma^{4}$ is required to reproduce the noise correctly ( $\Gamma$ is 
the lead-dot coupling energy, which contains the square of the tunneling elements $\mathbf{t}$ ). Finally, we consider the noise calculated numerically within the $2 \mathrm{vN}$ approach for two illustrative models. We compare our noise results for the single-resonant level (SRL) model with the exact results and demonstrate that, despite the limitations discussed above, the $2 \mathrm{vN}$ approach still gives a good description of the noise properties of this model for $\Gamma<k_{B} T$. We then investigate the noise and Fano-factor for the two-level spinless system where a canyon of current suppression was previously observed [20, 21].

The remainder of the paper is organized as follows: In Sec. 2 and Appendix A we present the $2 \mathrm{vN}$ approach with the inclusion of counting fields 19. Sec. 3 presents the proof, using Liouvillian perturbation theory, of the equivalence between the $2 \mathrm{vN}$ approach and the resonant tunneling approximation within the real-time diagrammatic technique. Sec. 4 gives a brief overview of the real-time diagrams employed in this work. Using diagrammatic techniques, we first show in Sec. 5 that the $2 \mathrm{vN}$ approach gives the correct EOM for the reduced density matrix of a single-level system coupled to reservoirs, but that this result does not generalize to the reduced density matrix $\rho$ of a multi-level many-body system. However, the EOM for the reduced single-particle density matrix is shown to exactly reproduced for non-interacting many-body systems, which allows for exact calculation of the current. In Sec. 6 it is shown that this does not hold for the noise, which cannot be reproduced exactly even in the non-interacting limit. Sections 7 and 8 contain the results of our $2 \mathrm{vN}$ calculations for the single-level system and the interacting two-level system. We then conclude in section 9

\section{The second order von Neumann method with counting fields}

A common transport Hamiltonian describes transitions (T) between two leads via a quantum dot (D) and can be written as

$$
\mathcal{H}=\mathcal{H}_{\text {Leads }}+\mathcal{H}_{\mathrm{D}}+\mathcal{H}_{\mathrm{T}} .
$$

We assume that the leads are Fermi liquids of electrons with dispersion $E_{k \ell}$. With $\ell$ we distinguish the different leads. Index $k$ is the (quasi-)momentum quantum number, but it could also contain additional properties like spin:

$$
\mathcal{H}_{\text {Leads }}=\sum_{k \ell} E_{k \ell} c_{k \ell}^{\dagger} c_{k \ell} \text {. }
$$

We describe the main system, i.e. the quantum dot, in its diagonal basis using the states $|a\rangle$ which can be many-particle states including also bosonic degrees of freedom:

$$
\mathcal{H}_{\mathrm{D}}=\sum_{a} E_{a}|a\rangle\langle a| .
$$

Responsible for transport are tunneling processes between the main system and the leads. These processes are described by terms where the state of the main system changes while at the same time an electron leaves or enters a reservoir. The tunneling Hamiltonian including counting fields, $\lambda_{\ell}$ with $\ell$ signifying at which lead electrons are counted, is given by 22

$$
\mathcal{H}_{T}(\lambda)=\sum_{k \ell, a b} T_{b a}(k \ell)|b\rangle\langle a| c_{k \ell} e^{-i \lambda_{\ell} / 2}+\text { h.c.. }
$$

$\ddagger$ When we refer to the order of a method, we refer to the power of $\Gamma$, and not that of the tunneling elements. 
In this section we use the convention that dot state $|b\rangle$ contains one more electron than dot state $|a\rangle$, and so on. In this formalism one can derive the generalized Liouvillevon-Neumann equation

$$
i \frac{d}{d t} \rho(\lambda, t)=\mathcal{H}^{+}(\lambda) \rho(\lambda, t)-\rho(\lambda, t) \mathcal{H}^{-}(\lambda),
$$

where, like in the rest of the paper, we set $\hbar=1$ for the sake of simplicity. We have introduced the $\lambda$-dependent Hamiltonian

$$
\mathcal{H}^{ \pm}(\lambda)=\mathcal{H}_{\text {Leads }}+\mathcal{H}_{\mathrm{D}}+\mathcal{H}_{\mathrm{T}}( \pm \lambda)
$$

In Laplace-space Eq. (5) reads

$$
i z \hat{\rho}(\lambda, z)=i \rho(\lambda, 0)+\mathcal{H}^{+}(\lambda) \hat{\rho}(\lambda, z)-\hat{\rho}(\lambda, z) \mathcal{H}^{-}(\lambda) .
$$

For states of the whole system we choose a basis of tensor products $|a g\rangle=|a\rangle \otimes|g\rangle$, where $|a\rangle$ describes the complete many particle state of the main system and $|g\rangle$ describes the many particle state of the leads.

We introduce the following quantities

$$
\begin{aligned}
& w_{b^{\prime} b}=\sum_{g} \rho_{b^{\prime} g ; b g}, \\
& \phi_{b a}(k \ell)=\sum_{g} \rho_{b g-k \ell ; a g}, \\
& \chi_{a b}(k \ell)=\sum_{g} \rho_{a g ; b g-k \ell}
\end{aligned}
$$

where $g-k \ell$ denotes a lead state where an electron with quantum number $k$ in lead $\ell$ has been removed. Note that $\chi_{a b}(k \ell) \neq \phi_{b a}^{*}(k \ell)$ due to the inclusion of counting fields. $w_{b^{\prime} b}$ are the elements of the reduced density matrix. In the EOM these elements couple to $\phi$ and $\chi$ which are the coherences arising from the superpositions of an electron in the leads and the dot. These in turn couple to elements where two electrons are moved between leads and dot. To break this hierarchy of equations the EOM is truncated as described below. This results in a closed EOM which can be solved numerically.

In order to achieve this we use the following approximations:

- Factorization and Thermodynamic limit: Wherever an occupation operator of a lead state appears we replace it by a Fermi function $f_{k \ell}$ of the respective lead. For example:

$$
\begin{aligned}
& \sum_{g}\left\langle a g\left|c_{k \ell}^{\dagger} c_{k \ell} c_{k^{\prime} \ell^{\prime}}^{\dagger} \hat{\rho}(\lambda, z)\right| b g\right\rangle \\
& \approx f_{k \ell} \sum_{g}\left\langle a g\left|c_{k^{\prime} \ell^{\prime}}^{\dagger} \hat{\rho}(\lambda, z)\right| b g\right\rangle
\end{aligned}
$$

This approximation is valid under the assumption that relaxation in the leads is quick, compared to the tunneling of individual $k$-states, so that equilibrium is restored between each tunneling event. This is well justified in the limit of an infinte number of lead states. Hence the occupations on the leads will not change due to the tunneling of single electrons, even not for large times. 
- Truncation: We neglect all matrix elements where the bra- and the ket-state differ by more than two single-particle states of the leads. For example:

$$
\begin{aligned}
& \left\langle a g\left|c_{k_{1} \ell_{1}} c_{k_{2} \ell_{2}}^{\dagger} c_{k_{3} \ell_{3}} \hat{\rho}(\lambda, z)\right| b g\right\rangle \approx 0 \\
& \text { if } k_{1} \ell_{1}, k_{2} \ell_{2} \text { and } k_{3} \ell_{3} \text { are different. }
\end{aligned}
$$

This represents neglection of higher order tunneling events, and is not valid in the regime $\Gamma \gg k_{B} T$.

Following the derivations in Refs. [17, 19] we can write down the EOM for $\phi, \chi$, and $w$, see Appendix A. Previously, implementations of this scheme have solved these equations numerically [17, 19] (and see section 7) but here we are interested in determining analytical properties of the approach. For this purpose the real-time diagrammatic technique [6, 7] is more suitable, as discussed in Sections 4 and 5.

\section{The 2vN method in Liouville space}

The structure of the $2 \mathrm{vN}$ scheme is most easily seen using the diagrams of Liouvillian perturbation theory (LPT) $22,24,25$. In particular, using this approach we demonstrate the equivalence of the $2 \mathrm{vN}$ scheme with the resonant-tunneling approximation. This approximation was introduced for the SRL in Ref. [26] and is defined in diagrammatic terms as retaining all irreducible diagrams in the kernel where a vertical cut crosses at most two lead contractions. Here we derive an explicit expression for the self-energy of the $2 \mathrm{vN}$ approach, which shows that the $2 \mathrm{vN}$ approach corresponds to exactly this approximation for an arbitrary system. We first discuss the theory without counting field, and include it at the end.

\subsection{Liouville space}

We begin by giving the essentials of LPT, but the reader is referred to Refs. 23, 24, 27, 25. for full details. The von Neumann equation for the evolution of the total density matrix under Hamiltonian Eq. (10) reads:

$$
\dot{\rho}(t)=-i[\mathcal{H}, \rho(t)]=\mathcal{L} \rho(t) .
$$

which defines the Liouvillian super-operator $\mathcal{L}$. In accordance with the decomposition of the Hamiltonian, $\mathcal{L}$ consists of three parts: $\mathcal{L}=\mathcal{L}_{\text {res }}+\mathcal{L}_{\mathrm{D}}+\mathcal{L}_{\mathrm{T}}$ with $\mathcal{L}_{\text {res }}=$ $-i\left[\mathcal{H}_{\text {Leads }}, \bullet\right], \mathcal{L}_{\mathrm{D}}=-i\left[\mathcal{H}_{\mathrm{D}}, \bullet\right]$, and $\mathcal{L}_{\mathrm{T}}=-i\left[\mathcal{H}_{\mathrm{T}}, \bullet\right]$. To ease book-keeping, we introduce a compact single index " 1 " to denote the triplet of indices $\left(\xi_{1}, k_{1}, l_{1}\right)$. The notation $\overline{1}$ refers to the triple $\left(-\xi_{1}, k_{1}, l_{1}\right)$. The first index $\xi_{1}= \pm$ describes whether a reservoir operator is a creation or annihilation operator:

$$
a_{1}=a_{\xi_{1} k_{1} \ell_{1}}= \begin{cases}c_{k_{1} l_{1}}^{\dagger}, & \xi_{1}=+ \\ c_{k_{1} l_{1}}, & \xi_{1}=-\end{cases}
$$

Similarly we define

$$
g_{1}= \begin{cases}\sum_{a b} T_{b a}^{*}(k l)|a\rangle\langle b|, & \xi_{1}=+ \\ \sum_{a b} T_{b a}(k l)|b\rangle\langle a|, & \xi_{1}=-\end{cases}
$$

such that the tunnel Hamiltonian can be written $\mathcal{H}_{\mathrm{T}}=\xi_{1} a_{1} g_{1}$, with all summations left implicit. The sign $\xi$ enters here because the reservoir operator always comes first in this expression for $\mathcal{H}_{\mathrm{T}}$, unlike in Eq. (4). 
The tunnel Liouvillian $\mathcal{L}_{\mathrm{T}}=-i\left[\xi_{1} a_{1} g_{1}, \bullet\right]$ consists of system and bath operators acting from both the left and the right. i.e. on both Keldysh branches. We introduce the superscript Keldysh index $p= \pm$ to describe these two possibilities and define corresponding super-operators in Liouville space. For the reservoir, we define $\mathcal{L}$-space super-operator $A$ via its action on the density operator $\rho$ :

$$
\left.A_{1}^{p}|\rho\rangle\right\rangle \leftrightarrow\left\{\begin{array}{ll}
a_{1} \rho, & p=+ \\
\rho a_{1}, & p=-
\end{array} .\right.
$$

Following Ref. 24], we define the system super-operators $G$ via

$$
G_{1}^{p} O=\sigma^{p} \times\left\{\begin{array}{cl}
g_{1} O, & p=+ \\
-O g_{1}, & p=-
\end{array} .\right.
$$

To avoid confusion we explicitly state the $G$ is thus not a Green's function. The object $\sigma^{p}$ is a dot-space super-operator with matrix elements

$$
\left(\sigma^{p}\right)_{s s^{\prime}, r r^{\prime}}=\delta_{s r} \delta_{s^{\prime} r^{\prime}}\left\{\begin{array}{cc}
1, & N_{s}-N_{s^{\prime}}=\text { even } \\
p, & N_{s}-N_{s^{\prime}}=\text { odd }
\end{array},\right.
$$

where, $N_{s}$ is the number of electrons in state $s$. The tunnel Liouvillian can then be written

$$
\mathcal{L}_{\mathrm{T}}=-i \xi_{1} p \sigma^{p} A_{1}^{p} G_{1}^{p}
$$

\subsection{The 2vN EOM in Liouville space}

Arranging the elements $w_{b^{\prime} b}$ into the vector $\left.|w\rangle\right\rangle$ and elements $\phi_{b a}\left(k_{1} l_{1}\right)$ into vector $|\phi(1)\rangle\rangle$ the $2 \mathrm{vN}$ EOM (without counting field), Eq. (A.1) and Eq. (A.2), read in Liouville space

$$
\begin{aligned}
z|w\rangle\rangle-|w\rangle\rangle\left(t_{0}\right)= & \left.\left.\mathcal{L}_{\mathrm{D}}|w\rangle\right\rangle+\sum_{1} T(1)|\phi(1)\rangle\right\rangle \\
z|\phi(1)\rangle\rangle= & \left.\left.\left(x_{1}+\mathcal{L}_{\mathrm{D}}\right)|\phi(1)\rangle\right\rangle+T_{f}(1)|w\rangle\right\rangle \\
& +\sum_{4} M_{f}(1,4) \phi(4),
\end{aligned}
$$

where, $T(1)=i G_{\overline{1}}^{p_{2}}$. We have also defined $T_{f}(1)=i p_{2} f\left(-\xi_{1} p_{2} \omega_{1}\right) G_{1}^{p_{2}}$ with Fermi function $f(\omega)=\left[e^{\beta \omega}+1\right]^{-1}$, and finally the block

$$
\begin{aligned}
& M_{f}(1,4)=-p_{3} f\left(-\xi_{3} p_{3} \omega_{3}\right) \times \\
& \left(\delta_{14} G_{\overline{3}}^{p_{2}} \frac{1}{z-x_{3}-x_{1}-\mathcal{L}_{\mathrm{D}}} G_{3}^{p_{3}}-\delta_{13} \delta_{42} G_{\overline{4}}^{p_{2}} \frac{1}{z-x_{4}-x_{1}-\mathcal{L}_{\mathrm{D}}} G_{1}^{p_{3}}\right),
\end{aligned}
$$

where summation over index 2 and 3 is implied, and where $x_{1}=-i \xi_{1}\left(\omega_{1}+\mu_{1}\right)$ with $\omega_{1}$ the energy of lead mode $\left(k_{1}, l_{1}\right)$ and $\mu_{1}$ the chemical potential of lead $l_{1}$. Diagrammatically, $M_{f}(1,4)$ is represented by:

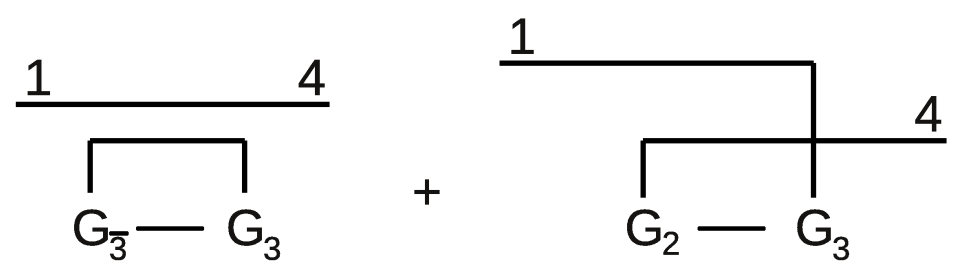

where symbol $G$ represents a tunnel vertex, the lines between the $G$ :s represent free propagation, and the lines on top indicate contraction of lead operators. 


\subsection{The $2 v N$ self-energy}

An expression for the system self-energy within the $2 \mathrm{vN}$ method can be derived by iterating Eq. (19). Defining the free system propagator $\Omega(1)=\left[z-x_{1}-\mathcal{L}_{\mathrm{D}}\right]^{-1}$ we obtain

$$
\begin{aligned}
& \left.\left.|\phi(1)\rangle\rangle=\Omega(1)\left\{T_{f}(1)|w\rangle\right\rangle+\sum_{2} M_{f}(1,2)|\phi(2)\rangle\right\rangle\right\} \\
& \left.\left.\left.=\Omega(1) T_{f}(1)|w\rangle\right\rangle+\Omega(1) \sum_{2} M_{f}(1,2)\left\{\Omega(2) T_{f}(2)|w\rangle\right\rangle+\Omega(2) \sum_{3} M_{f}(2,3)|\phi(3)\rangle\right\rangle\right\} \\
& \left.\left.=\Omega(1) T_{f}(1)|w\rangle\right\rangle+\Omega(1) M_{f}(1,2) \Omega(2) T_{f}(2)|w\rangle\right\rangle \\
& \left.+\Omega(1) M_{f}(1,2) \Omega(2) M_{f}(2,3) \Omega(3) T_{f}(3)|w\rangle\right\rangle+\ldots
\end{aligned}
$$

Substituting into Eq. (18) and solving gives

$$
\left.|w(z)\rangle\rangle=\frac{1}{z-\mathcal{L}_{\mathrm{D}}-\Sigma(z)}\left|w\left(t_{0}\right)\right\rangle\right\rangle
$$

with the (non-Markovian) self-energy

$$
\begin{aligned}
\Sigma(z)= & T(1) \Omega(1) T_{f}(1)+T(1) \Omega(1) M_{f}(1,2) \Omega(2) T_{f}(2) \\
& +T(1) \Omega(1) M_{f}(1,2) \Omega(2) M_{f}(2,3) \Omega(3) T_{f}(3)+\ldots
\end{aligned}
$$

with summation over all indices implied. This we can write in a compact form. Let $\mathbf{T}$ be a vector with elements $T(1), \mathbf{T}_{f}$ be a vector with elements $T_{f}(1)$. Furthermore let $\mathbf{M}$ be the matrix with elements $M_{f}(1,2)$ and finally, $\boldsymbol{\Omega}$ be a diagonal matrix with elements $\Omega(1) \delta_{1,2}$. The self-energy can then be written

$$
\begin{aligned}
\Sigma(z)= & \mathbf{T} \cdot \boldsymbol{\Omega} \cdot \mathbf{T}_{f}+\mathbf{T} \cdot \boldsymbol{\Omega} \cdot \mathbf{M} \cdot \boldsymbol{\Omega} \cdot \mathbf{T}_{f} \\
& +\mathbf{T} \cdot \boldsymbol{\Omega} \cdot \mathbf{M} \cdot \boldsymbol{\Omega} \cdot \mathbf{M} \cdot \boldsymbol{\Omega} \cdot \mathbf{T}_{f}+\ldots,
\end{aligned}
$$

which can clearly be resummed to give

$$
\Sigma(z)=\mathbf{T} \cdot \frac{1}{1-\boldsymbol{\Omega} \cdot \mathbf{M}} \cdot \boldsymbol{\Omega} \cdot \mathbf{T}_{f}
$$

which is a very nice clean result.

This self-energy can be expanded in terms of the diagrams of Liouville perturbation theory, see Refs. [23, 28, 25. For example, the product of $\mathbf{M} \boldsymbol{\Omega} \cdot \mathbf{M}$ entering in Eq. (23) formally corresponds to the diagrammatic expression,

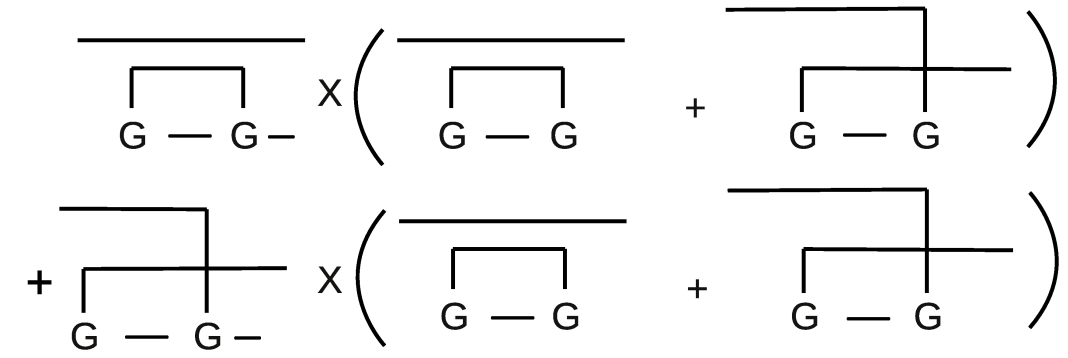

where all indices have been suppressed. When diagrams are multiplied unpaired contraction lines are connected. As each diagram in $\mathrm{M}$ only has one unpaired 
contraction line it is ensured that there will never be more than two contraction lines at any time. By including the terminations $T$ and $T_{f}$ in Eq. (23) and remaining free propagators, one arrives at the diagrammatic expression for the self energy

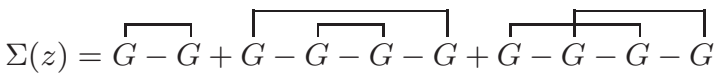

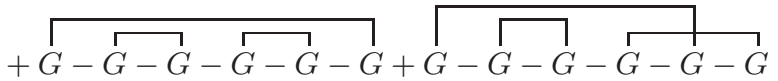

$$
\begin{aligned}
& +\underset{+G-G-G-G-G}{+G-G-G-G-G-G+\ldots,}
\end{aligned}
$$

From this expansion it is clear that the $2 \mathrm{vN}$ self-energy contains the set of all diagrams in which there are at most two contraction lines passing over any given point. This exactly proves the equivalence between the $2 \mathrm{vN}$ approach and the resonant tunneling approximation.

Finally we define the propagator in Laplace space

$$
\rho(\omega)=\Pi(\omega) \rho\left(t_{0}\right)=\frac{1}{-i \omega+\mathcal{L}_{\mathrm{D}}+\Sigma(-i \omega)} \rho\left(t_{0}\right),
$$

which will be used in Sec. 6 .

\subsection{Counting statistics}

Counting fields can easily be added in the kernel Eq. (25) by replacing the bare tunnel vertices with their gauge-transformed counterparts: $G_{1}^{p_{1}} \rightarrow G_{1}^{p_{1}} e^{i \xi_{1} p_{1} \lambda_{\ell_{1}} / 2}$ [28. In the exponent here, $\xi_{1}$ corresponds to whether an electron is created or annihilated (see just before Eq. (12)) and $p_{1}$ is the Keldysh index. With this replacement, the non-Markovian $\lambda$-dependent $2 \mathrm{vN}$ self-energy then reads

$$
\Sigma(\lambda ; z)=\mathbf{T}(\lambda) \cdot \frac{1}{1-\boldsymbol{\Omega} \cdot \mathbf{M}(\lambda)} \cdot \mathbf{\Omega} \cdot \mathbf{T}_{f}(\lambda)
$$

with matrices

$$
\begin{aligned}
& T(\lambda ; 1)=i G_{\overline{1}}^{p_{2}} e^{i \xi_{1} p_{1} \lambda_{\ell_{1}} / 2} \\
& T_{f}(\lambda ; 1)=i p_{2} f\left(-\xi_{1} p_{2} \omega_{1}\right) G_{1}^{p_{2}} e^{i \xi_{1} p_{1} \lambda_{\ell_{1}} / 2} \\
& M(\lambda ; 1,4)= \\
& -p_{3} f\left(-\xi_{3} p_{3} \omega_{3}\right)\left\{\delta_{14} G_{\overline{3}}^{p_{2}} \Omega(1+3) G_{3}^{p_{3}} e^{i \xi_{3}\left(p_{3}-p_{2}\right) \lambda_{\ell_{3}} / 2}\right. \\
& \left.-\delta_{13} \delta_{4} G_{\overline{4}}^{p_{2}} \Omega(1+4) G_{1}^{p_{3}} e^{-i \xi_{4} p_{2} \lambda_{\ell_{4}} / 2} e^{i \xi_{1} p_{3} \lambda_{\ell_{1}} / 2}\right\}
\end{aligned}
$$

where e.g. $\Omega(1+4)$ denotes the free propagator $\left[z-x_{1}-x_{4}-\mathcal{L}_{\mathrm{D}}\right]^{-1}$.

Derivatives of $\Sigma(\lambda ; z)$ with respect to $\lambda$ then give the FCS. The current and finite-frequency noise can be written in a "current block" notation as 28, 29,

$$
\begin{aligned}
\langle I\rangle & =e\left\langle\left\langle J_{1 a}(0)\right\rangle\right\rangle, \\
S(\omega) & =\frac{e^{2}}{2}\left\langle\left\langle J_{2 a}(\omega) \rho_{s t}+J_{1 a}(\omega) \Pi(\omega) J_{1 b}(\omega)\right\rangle\right\rangle \\
& -2 \pi \delta(\omega)\langle I\rangle^{2}+\text { terms with }(\omega \rightarrow-\omega),
\end{aligned}
$$

where $\langle\langle\ldots\rangle\rangle=$ denotes expectation with respect to the stationary state of the system and where $J_{1 a}, J_{1 b}$, and $J_{2 b}$ are current blocks (see Appendix Appendix B for explicit forms). The diagrams contributing to $J_{1 a}(\omega)$ and $J_{1 b}(\omega)$ are equivalent to those 
contributing to the self energy $\Sigma(z=-i \omega)$ except that one tunneling vertex has been replaced by a current vertex. In $J_{1 a}(\omega)$ the leftmost vertex has been replaced, while in $J_{1 b}(\omega)$ any replacement is possible. Such a replacement changes the value of a diagram by a factor of $1 / 2$ and furthermore introduces a sign change if the current vertex is moved to the other branch. We also define $J_{2 a}(\omega)$ where two tunneling vertices have been replaced by current vertices. One of the current vertices must be to the left, the other can be at any position. The reason that $J_{1 a}$ and $J_{2 a}$ needs a current vertex at the leftmost position is that when the trace is taken and the Keldysh contour closed all diagrams with a tunneling vertex to the left will cancel each other.

\section{Diagrammatic description}

Our aim is now to investigate when and why the $2 \mathrm{vN}$ approach is exact. For this purpose the LPT used in the previous section is too compact. We can, however, use the result of the previous section that the $2 \mathrm{vN}$ approximation is equivalent to the resonant tunneling approximation within the real time diagrammatic technique. From here on we will therefore only use two-branch Keldysh diagrams (which the LPT diagrams are summations over). The upper and lower contour of the Keldysh diagram give the time evolution of the bra- and ket-states respectively. This corresponds to different ordering on the contours so that the contour ordering of the tunneling events agrees with the direction of time along the upper branch, while it is opposite to the direction of time on the lower branch. One can view this as the time running backwards along the lower branch. This section is devoted to a discussion on how the EOM for the reduced density matrix of a central quantum system coupled to external leads can be derived using such diagrammatic methods.

In deriving the EOM we follow the diagrammatic notation of Ref. [12, where more details on how the diagrams are evaluated can be found. Assuming that the total density matrix is a direct product of the initial states of the dot and leads at the time $t_{0}$, when the couplings between leads and dot is switched on, the EOM for the elements of the reduced density matrix $\rho_{b ; b^{\prime}}$ can be written as a closed set of linear equations

$$
\begin{aligned}
\frac{d \rho_{b ; b^{\prime}}}{d t}= & -i\left(E_{b}-E_{b^{\prime}}\right) \rho_{b ; b^{\prime}} \\
& +\sum_{a a^{\prime}} \int_{t_{0}}^{t} d \tau K_{b b^{\prime}}^{a a^{\prime}}(t-\tau) \rho_{a ; a^{\prime}}(\tau),
\end{aligned}
$$

where the first term on the right corresponds to the unitary evolution of the dot disregarding couplings to leads and the second term is generated by tunneling events. Unlike in Sec. 2 it is not assumed that state $|b\rangle$ contains one more electron than state $|a\rangle$. Here the coefficients $K_{b b^{\prime}}^{a a^{\prime}}$ can be calculated using Keldysh diagrams as outlined in Ref. [12. All possible irreducible diagrams connecting the states $|b\rangle,\left|b^{\prime}\right\rangle$ on the left with the states $|a\rangle,\left|a^{\prime}\right\rangle$ on the right contribute to the coefficient $K_{b b^{\prime}}^{a a^{\prime}}$. In an irreducible diagram any vertical cut crosses at least one lead contraction arrow. We use the following notation $|b\rangle=|\alpha, \beta, \ldots\rangle$, where anti-symmetrization of the manyparticle state is implicitly assumed, to specify which single-particle states $|\alpha\rangle,|\beta\rangle, \ldots$ that compose the many-body state $|b\rangle$. This way of writing the many-particle state as product states is generally not possible for interacting systems but can always be done in the non-interacting case that we focus on. 
Here we give a brief summary of the diagrammatic rules, using an example diagram giving the time evolution on $\rho_{1,2,3 ; 1,2,3}$ in terms of $\rho_{1 ; 1}$, see Fig. 1. More detailed discussions can be found in Refs. [6, 7, 12, Throughout the paper we use the convention that time increases from right to left.

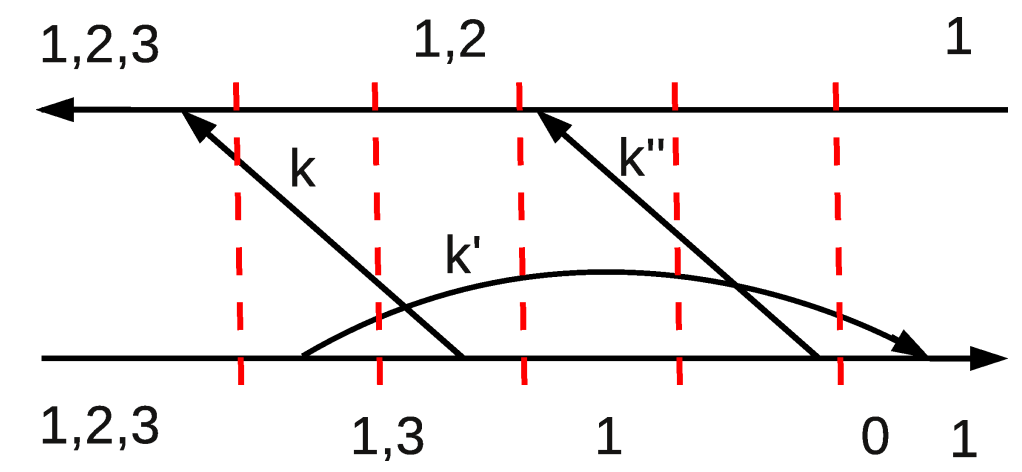

Figure 1. Example of a 3:rd order diagram included in the $2 \mathrm{vN}$ method.

1) Each vertex where a lead contraction arrow ends has a related tunneling probability $T_{b a}(k l)$, representing that the dot state changes from many-particle state $|a\rangle$ to many-particle state $|b\rangle$ by the annihilation of an electron in lead-state $k$ in lead $l$. Arrows pointing out of a branch correspond to Hermitean conjugated processes.

2) In a contraction, the lead operator with the larger time argument comes first unless the right vertex of the contraction is on the lower branch. For Fig. 1 1 this results in the Fermi factors: $f\left(E_{k}\right)\left[1-f\left(E_{k^{\prime}}\right)\right] f\left(E_{k^{\prime \prime}}\right)$, where we have included the lead index $\ell$ in $k$.

3) The factors in the denominator of the diagram are given by the dashed vertical lines of Fig. 1. Each line gives a factor which is given by (the energy on the lower contour) - (the energy on the upper contour) + (energies of arrows pointing right) (energies of arrows pointing left). Furthermore $i 0^{+}$should be added to each factor.

4) The sign of the diagram is given by $(-1)^{N+n_{c}+n_{l}} \times($ Fermion sign), where $N$ is the order of the diagram in $\Gamma, n_{c}$ is the number of crossings of contraction lines, and $n_{l}$ is the number of vertices on the lower contour. In Fig. 1 $N=3, n_{c}=2$, and $n_{l}=4$ so that the first sign factor becomes -1 . Comparing with Ref. [12] the Fermion sign results from the ordering of the dot operators. For the diagram in Fig. 1 the Fermion sign is given by $a_{1}^{\dagger} a_{1} a_{3} a_{2} a_{3}^{\dagger} a_{2}^{\dagger}|1\rangle=-|1\rangle$, i.e. the Fermion sign is negative, resulting in an overall positive sign in front of the diagram.

\section{Cancellation of diagrams for non-interacting systems}

In this Section we show how all diagrams discarded in the resonant tunneling approximation, i.e. the diagrams not included in the $2 \mathrm{vN}$ approach, cancel each other in the EOM for the single-particle reduced density matrix in the non-interacting limit.

The single-particle reduced density matrix is defined as

$$
p_{\mu ; \nu}=\operatorname{Tr}_{\operatorname{dot}}\left[a_{\nu}^{\dagger} a_{\mu} \rho\right]
$$

where the trace is taken over the dot system. $\rho$ and $p$ are related to each other in the following way: The diagonal states of $\rho$ give the probability of the corresponding 
many-body state being occupied. This probability corresponds to that exactly the single-particle states that compose the many body state are occupied, the others are empty. The diagonal terms of $p$ give the probability that the corresponding singleparticle state is occupied, without considering the occupations of the other singleparticle states.

The exact EOM for the single-particle reduced density matrix of non-interacting systems is a major strength of the $2 \mathrm{vN}$ approach as important physical observables such as the current can be exactly calculated from this knowledge.

For single level QDs a canceling partner diagram can always be found for each diagram by changing the branch of the second and third leftmost vertices in the diagram, see Figs. 2] and 3. For multi-level QDs the diagrams can either be canceled by methods similar to those used for the single level QDs, or be grouped into families, see Fig. 5 where all diagrams have the same absolute value but half contribute to the EOM with plus signs and half with minus signs.

\subsection{Single resonant level}

To explain on a diagrammatic basis why the resonant tunneling approximation gives the right $\mathrm{EOM}$ for the single-particle reduced density matrix and the right current we consider diagrams with three dot-lead excitations at the same time. We begin by noting that diagrams where different vertices are contracted necessarily have different denominators. When trying to find out how the diagrams cancel a good strategy is therefore to look for diagrams where the same vertices are contracted, i.e. diagrams that correspond to the same Liouvillian diagram (see Sec. 3). Since it is a single-level quantum dot, we are limited to occupations of $|0\rangle$ or $|1\rangle$. This means that at every second vertex we must create an electron in the dot while the other vertices must annihilate an electron. Specifically this means that if we create at vertex 4 we must do the same at vertex 3 if they are on different branches, and if we create at 3 we must annihilate at 4 if they are on the same branch.

A canceling diagram can be found for any third-order diagram not included in the $2 \mathrm{vN}$ approach by changing branch of vertex 3 and 4 . This results in the same change of occupation at the two branches so that the energy denominators are unchanged. As we only moved the left vertices in the contraction, the contributions from the lead contractions (Fermi factors) are also unchanged. Since we are dealing with a single-particle system, we can neglect the Fermion sign and the total sign is given by $(-1)^{N+n_{c}+n_{l}}$. Clearly $N$ is unchanged. The change in $n_{l}$ is 0 or \pm 2 , i.e. an even number. The change in $n_{c}$ is given by the number of dot operators that are commuted. The two moved vertices will both be commuted with the unmoved left vertex 5 . Then there is the additional commutation between the two moved vertices. In total $n_{c}$ is therefore an odd number.

We will illustrate the canceling using an example diagram seen in Fig. 2. The red numbers on gray backgrounds give the state of the quantum dot, while the black numbers label the vertices. Changing branch of vertex 3 and 4 for the diagram in Fig. 2 results in the cancelling partner diagram shown in Fig. 3.

For higher-order diagrams the reasoning is much the same. A canceling diagram can again be found by moving the second and third leftmost vertices to the other branch. In the same way as for the third-order diagrams the change in number of crossings is an odd number so that the diagrams cancel. This explains on a diagrammatic basis why the $2 \mathrm{vN}$ method gives the exact $\mathrm{EOM}$ for $\rho$ and the exact 


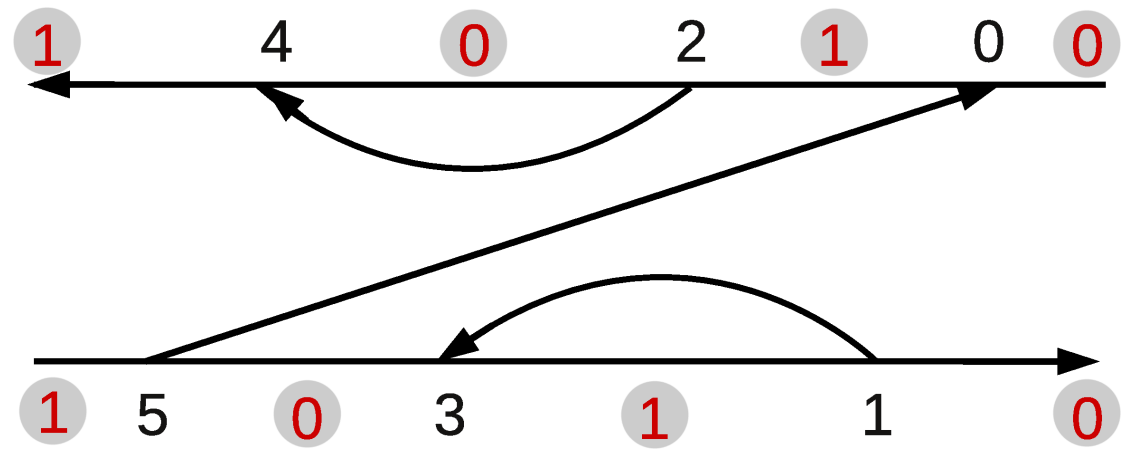

Figure 2. Example of a 3:rd order diagram, for a single level system, that is not included in the $2 \mathrm{vN}$ approach. The red numbers on gray backgrounds denote the dot occupation while the black numbers label the indices.

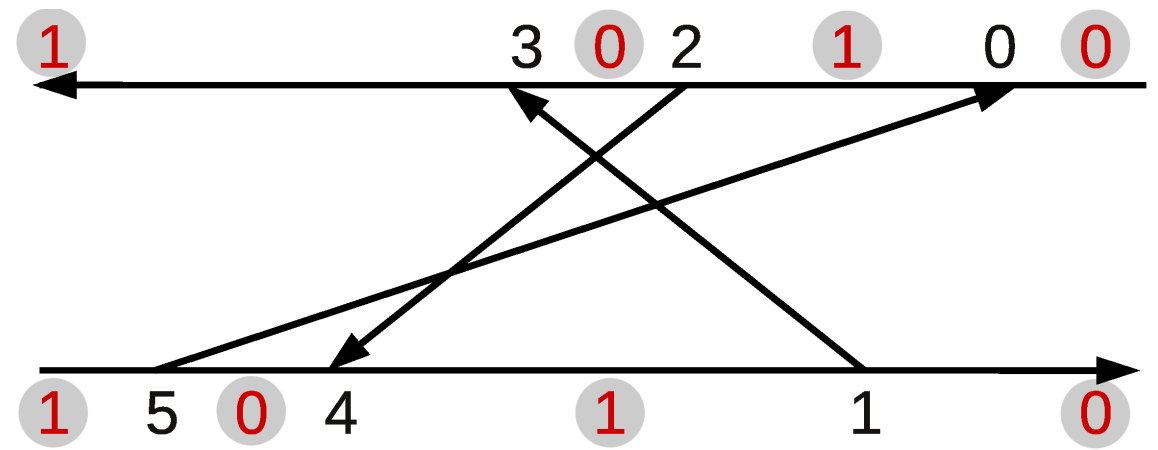

Figure 3. Canceling partner of the diagram in Fig 2

current for the single level 17 .

The above described method explains how to cancel diagrams where three dotlead excitations exist at the same time, i.e. the type of diagrams not included in the $2 \mathrm{vN}$ approach. We emphasize that higher order diagrams where the maximum number

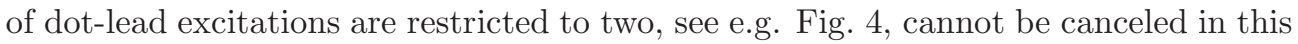
way. Changing branch of the same vertices as before, in Fig. 4 labeled by 2 and 4 since the vertices have been shifted in time, changes the number of crossings in the diagram with an even number. As a result the two diagrams do not cancel. This demonstrates the improvement of the $2 \mathrm{vN}$ approach compared to pure second order approaches where diagrams such as Fig. 4 are not included.

\subsection{Canceling of diagrams for multi-level systems}

For multiple level QDs it is generally not possible to cancel the diagrams in the above described way. The reason is that there is no requirement that electrons are created/annihilated at every second vertex as many different states in the dot can be active. In order for the diagrams to cancel the two moved vertices must create/annihilate the same state in the dot. Generally it is not possible to find two such vertices in many-body diagrams. As a result, the $2 \mathrm{vN}$ approach does not 


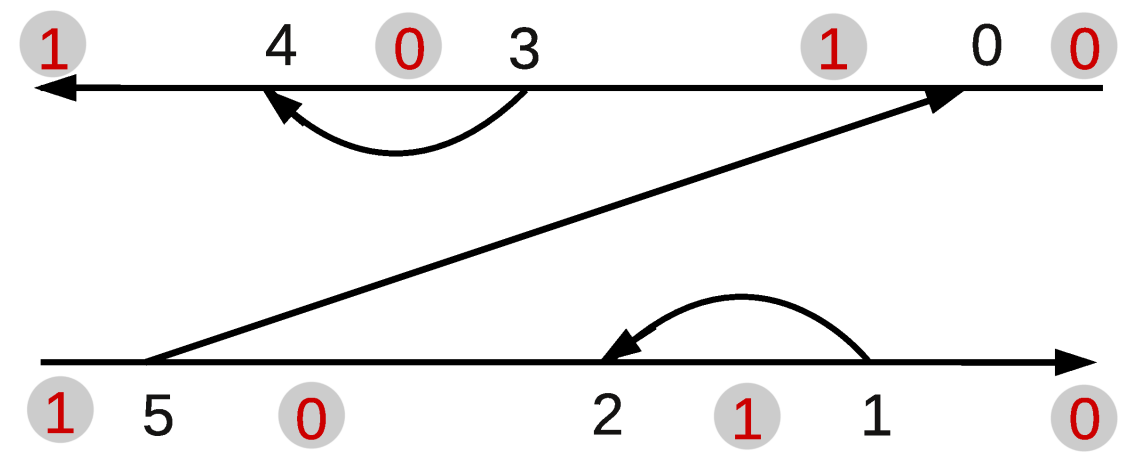

Figure 4. Example of a higher-order diagram included in the $2 \mathrm{vN}$ approach that is not canceled by the above described method.

give the correct EOM for $\rho$ for many-body systems even in the noninteracting limit. Importantly, we will see that the EOM for the single-particle reduced density matrix is still correct in this limit.

Following the definition of the single-particle reduced density matrix $p$ we have for non-interacting systems, where the many-particle states always can be written as direct products of single-particle states:

$$
p_{\mu ; \nu}=\sum_{c:(\mu, \nu \notin c)} \rho_{\mu+c ; \nu+c} .
$$

Here, the state $\mu+c$ is the many-particle state $c$ with a particle added in single-particle state $\mu$. To get the time evolution of $p_{\mu ; \nu}$ in terms of $\rho_{a ; a^{\prime}}$ we must therefore sum over all diagrams giving the time evolution of $\rho_{\mu+c ; \nu+c}$ in terms of $\rho_{a ; a^{\prime}}$. In this sum many of the diagrams will have the same energy denominators and Fermi factors, i.e. the values of the diagrams are equal, which means that they cancel if they have opposite sign.

Before continuing we introduce the concept of free vertices: The free vertices of a diagrams are those among the $N$ left vertices where states other than $\mu$ and $\nu$ are active. To explain how the canceling works we divide all diagrams of third order and higher into four groups:

1) No state occurs more than once among the free vertices.

2) Some states occur twice among the free vertices.

3) Some states occur three times or more among the free vertices.

4) There are no free vertices. This can only happen if $\mu \neq \nu$.

It is clear that any diagram falls into one of theses groups. Below we explain how the canceling works for each group.

Group 1: We will see that a family of diagrams can be constructed by changing branch of the left vertices where states other than $\mu$ or $\nu$ are active. It is clear that every diagram belongs to one and only one family. Moving the vertices in such a way does not affect the energy denominators of the diagram, and since only the left vertices are moved the contributions from the Fermi-factors are also unaffected. The diagrams within a family will thus cancel if equal numbers contribute with plus and minus sign. It should be noted that the energy denominators of the different diagrams in a family are only equal in the non-interacting limit. For interacting systems the diagrams do not cancel. 
The number of free vertices will be denoted by $n$ and the number of these that are on the upper branch will be denoted by $k$. The number of diagrams in a family with $k$ out of the $n$ free vertices on the upper branch is then given by $\left(\begin{array}{l}n \\ k\end{array}\right)$.

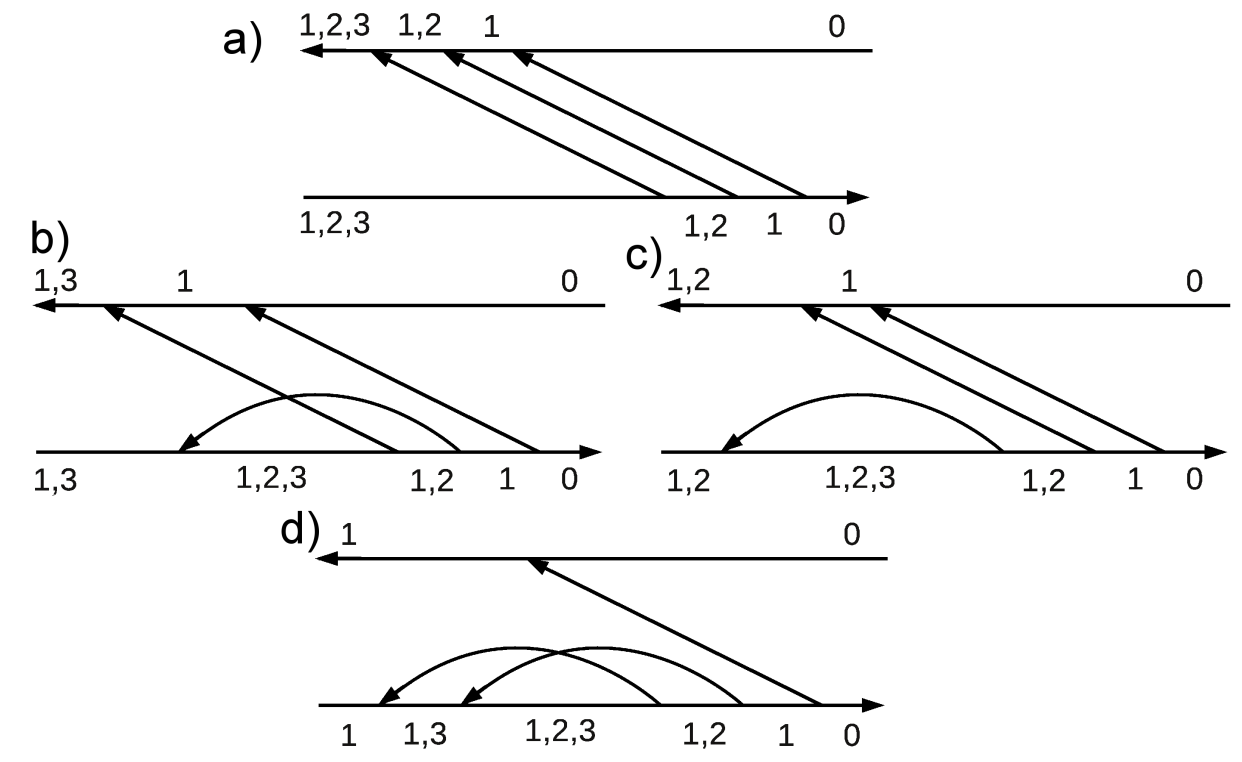

Figure 5. A family of canceling diagrams in group 1.

We first show that all diagrams with a given $k$ have the same sign. In the expression $(-1)^{N+n_{c}+n_{l}}$ the number of crossings between contraction lines changes when we move one vertex from the upper to the lower and one from the lower to the upper. However, this is canceled by the additional change in the Fermion sign, as each change in the number of crossings is related to the commutation of two Fermion operators of different dot states. In total there will therefore not be a sign change. Next we show that the sign changes when $k$ is changed by one. Moving a vertex can result in a change in the number of crossing contraction lines, but this number is again the same as the number of fermion operators that is commuted due to the change. Left is only the change in sign due to that the number of vertices on the lower branch has changed by one. In the general case one therefore gets a sum

$$
\sum_{k=0}^{n}(-1)^{k}\left(\begin{array}{l}
n \\
k
\end{array}\right)=0 \text { for } \mathrm{n} \geq 1
$$

Since each diagram belongs to one and only one family and each family gives no contribution we conclude that this group of diagrams gives no contribution.

An example family of diagrams belonging to group 1 is shown in Fig. 5 . Here, $|\mu\rangle=|\nu\rangle=|1\rangle$, and on the right we have the state $|0\rangle$. This family of diagrams thus contribute to the time evolution of $p_{1 ; 1}$ in terms of $\rho_{0 ; 0}$. For this family of diagrams $n=2$, corresponding to the states $|2\rangle$ and $|3\rangle$.

Group 2: When some states occurs twice among the free vertices only the leftmost of these two vertices can be moved to the other branch. If the right of these vertices is moved, a state will be created or annihilated twice in a row. However, all diagrams in this group have at least one vertex that can be moved to the other branch which 
results in a family of canceling diagrams according to Eq. (35). An example of such a diagram is shown in Fig. 6. Moving the right vertex marked with a red square results in that state $|2\rangle$ is annihilated twice in a row.

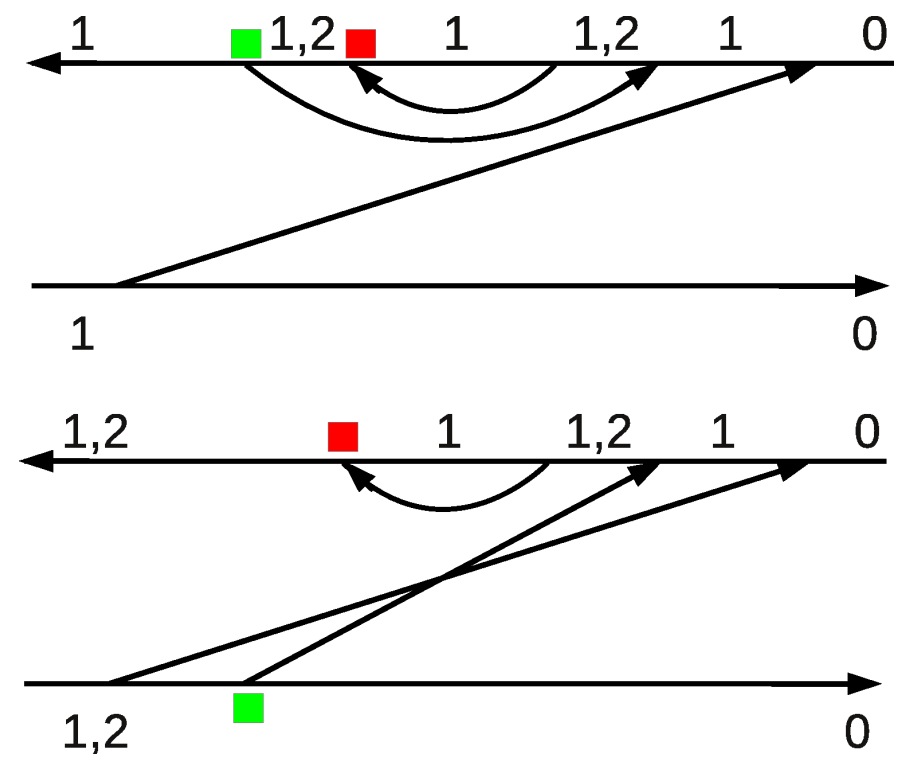

Figure 6. A family of canceling diagrams in group 2. Only the vertex marked with a green square can be moved to the other branch.

Group 3: If the same state $|\alpha\rangle$ occurs three times or more among the free vertices the diagram can be canceled by exactly the method used for single-level diagrams. When moving the vertices corresponding to state $|\alpha\rangle$ crossings with contraction lines for other states will occur. However, this sign change is canceled by the commutation of Fermion operators as described above. Vertices and contraction lines not belonging to $|\alpha\rangle$ can therefore be neglected which results in a single level diagram in state $\alpha$.

Group 4: Diagrams with no free vertices can for $N \geq 3$ only occur if $\mu \neq \nu$. If there are no free vertices in the diagram it means that no states other than $\mu$ or $\nu$ occur in the diagram. Thus at least two of the $N$ vertices to the left must act on the same state. These two vertices must necessarily be on the same branch and one creates the state while the other annihilates the state. If there is no creation vertex of the same state on the other branch to the left of these two vertices, a canceling diagram is found by changing branch of the two vertices, see Fig. 7 a). In the other case one changes branch on the two creation vertices, see Fig. (7b). Fig. 7 only shows the two possibilities for the three left vertices as the cancelling of the diagrams can be explained without looking at the right part of the diagram. Moving the vertices like this works in the same way as for single level diagrams. It results in two diagrams with opposite sign but equal Fermi-factors and energy denominators.

To conclude one can divide all diagrams into those that cancel directly for $\rho$, and those that cancel only for $p$. The ones that cancel directly for $\rho$ can be canceled by moving two vertices in a way that does not change $b$ and $b^{\prime}$. To cancel the rest of the diagrams for $p$ the diagrams are divided into families. All diagrams within a family contributes to the time evolution of the same element in $p$. All diagrams in the family 


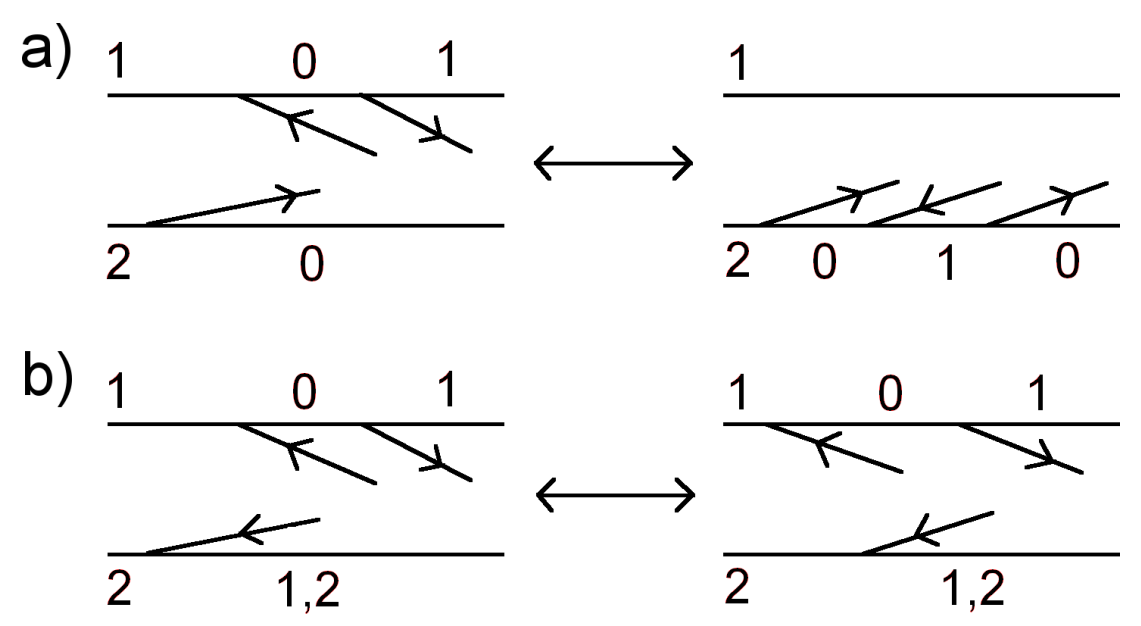

Figure 7. Demonstration of how diagrams belonging to group 4, i.e. diagrams with no free vertices, are canceled.

have the same value but half contribute with a plus sign and half with a minus sign. As a result of the exact EOM for $p$, the current can be calculated exactly for any non-interacting system, as outlined in Ref. [18.

It is interesting to note that although the $2 \mathrm{vN}$ method does not correctly reproduce $\rho$, it can in some cases still be calculated exactly using the knowledge that the EOM for $p$ is exact. For systems where the probability to tunnel into superpositions of the dot states can be neglected, such as the non-interacting Anderson model or dots where the level spacing greatly exceeds the coupling strengths so that the secular approximation can be performed, each dot level can be treated independently from the others. This allows us to write down the following expression for the diagonal elements of $\rho$,

$$
\rho_{b ; b}=\prod_{\alpha \in b} p_{\alpha ; \alpha} \prod_{\beta \notin b}\left(1-p_{\beta ; \beta}\right),
$$

where the first product is over all single-particle states composing $b$ and the second product is over those not belonging to $b$. For such systems the $2 \mathrm{vN}$ method thus allows for an exact derivation of the $\mathrm{EOM}$ also for $\rho$. We illustrate how this works for the non-interacting Anderson model. Here the elements of $\rho$ can be calculated from $p$ as

$$
\begin{aligned}
& \rho_{0 ; 0}=\left(1-p_{\uparrow ; \uparrow}\right)\left(1-p_{\downarrow ; \downarrow}\right), \\
& \rho_{\uparrow ; \uparrow}=p_{\uparrow ; \uparrow}\left(1-p_{\downarrow ; \downarrow}\right), \\
& \rho_{\downarrow ; \downarrow}=\left(1-p_{\uparrow ; \uparrow}\right) p_{\downarrow ; \downarrow}, \\
& \rho_{d ; d}=p_{\uparrow ; \uparrow} p_{\downarrow ; \downarrow},
\end{aligned}
$$

where $\rho_{d ; d}$ denotes the doubly occupied state. We again remark that this is only possible for non-interacting systems where the occupations of the different single particle levels are independent. 


\section{Calculating the noise for single resonant level systems}

To investigate if the noise is exact we will study the constituting parts of Eq. (31). From the above discussion it is evident that the time evolution operator in Laplace space $\Pi(\omega)$, Eq. (27), is correctly reproduced by the $2 \mathrm{vN}$ approach for single-particle systems. Furthermore the diagrams in the $J_{1 a}$ block of Eq. (31) excluded from the $2 \mathrm{vN}$ scheme can be canceled by the same method as for single-particle systems.

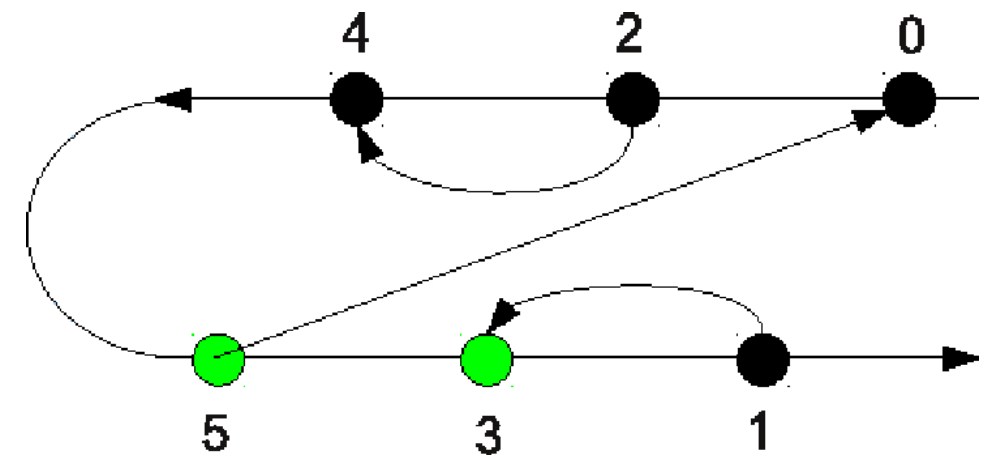

Figure 8. Example of a 3:rd order noise diagram for $J_{2 a}$ which cannot be canceled by the above described method. The green dots correspond to current vertices while the black represent normal tunneling vertices. The trace in Eq. (31) is represented by the closing of the Keldysh contour.

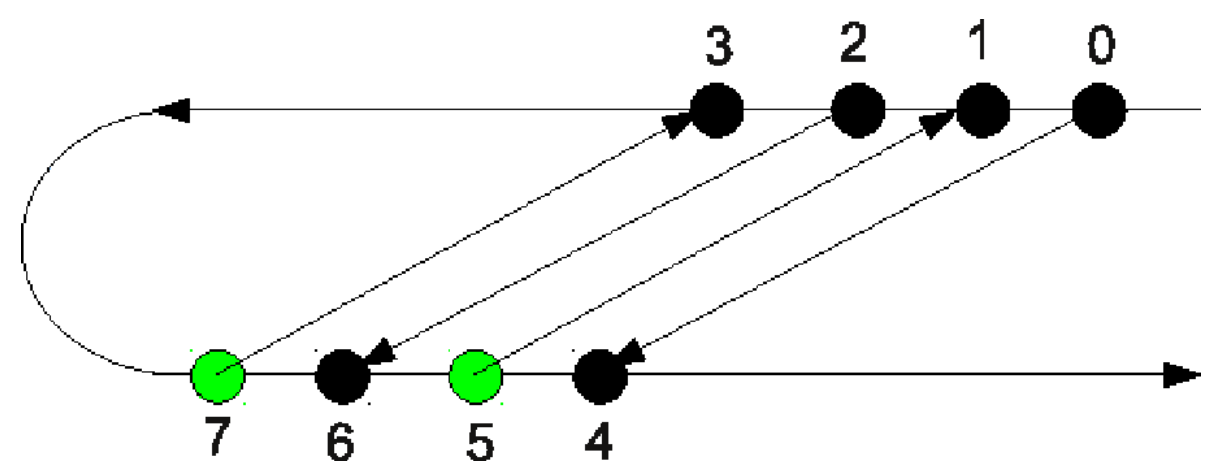

Figure 9. Example of a 4 :th order noise diagram for $J_{2 a}$ which cannot be canceled by the above described method. The green dots correspond to current vertices while the black represent normal tunneling vertices. The trace in Eq. 311 is represented by the closing of the Keldysh contour.

However, the current vertex in blocks $J_{1 b}$ and $J_{2 a}$ that occurs at positions other than leftmost prevents the canceling of diagrams. Moving such a vertex to the other branch results in an additional sign change so that the diagrams add upp instead of cancelling. This is illustrated in Fig. 8 which cannot be canceled by moving vertex 3 . Thus, this 3:rd order diagram is likely to contribute to the noise.

There are also 4:th order diagrams that contribute to the shot noise, see e.g. Fig. 9 . 
It should be noted that although the counting statistics was introduced for finite frequency noise, the results also holds at zero frequency so that the noise cannot be correctly calculated by the $2 \mathrm{vN}$ approach in this limit.

Going to fifth order or higher, one can always find two normal tunnel vertices that can be moved without resulting in a diagram with double occupation. 4:th order in $\Gamma$ is thus required to calculate the shot noise exactly for the single level. Each order of cumulants in the FCS adds an additional current vertex. As a result one needs to include two more orders of diagrams, which quickly results in great numerical efforts being required.

It should be pointed out that we have not presented a proof that the 3:rd and 4:th order noise diagrams do not cancel. Rather we have shown that the method by which the diagrams for the EOM cancel, does not work for the noise diagrams. It could be argued that the noise diagrams cancel by some other manner, but previous analytical work have shown that the $2 \mathrm{vN}$ method does not reproduce the noise exactly for the single resonant level [19. That 4:th order diagrams are required is also expected from the transmission formula, see Ref. 1. In the low-temperature limit the noise is proportional to $T(1-T), T$ being the transmission function. Here the $T^{2}$ term is proportional to $\Gamma^{4}$. It is interesting to note that although the $2 \mathrm{vN}$ approach does not give the correct noise, it can still be obtained by inserting the correct transmission function from the $2 \mathrm{vN}$ method into the transmission formula. In the non-interacting limit the transmission function can be obtained from the current as it does not depend on the applied voltage.

\section{The single resonant level}

Having seen that higher-order terms are required to correctly reproduce the noise of single-particle systems it is of interest to see how large the discrepancy is between the $2 \mathrm{vN}$ method and the exact results using the transmission formula.

To get an impression of this discrepancy, we apply the $2 \mathrm{vN}$ approximation to the noise in the single resonant level model.

Assuming the (constant) tunneling rates $\Gamma_{\alpha}=2 \pi \sum_{k}\left|t_{k \alpha}\right|^{2} \delta\left(E-E_{k \alpha}\right)$ and $\Gamma=\Gamma_{L}+\Gamma_{R}$, and defining the quantity $B_{\ell}(E) \equiv 2 \pi \sum_{k} t_{k \ell}^{*} \phi_{10}(z, \lambda ; k \ell) \delta\left(E-E_{k \ell}\right)$, we obtain the EOM for the single resonant level

$$
\begin{aligned}
& i z w_{00}(z, \lambda)-i w_{00}(t=0, \lambda)=\sum_{\ell} e^{i \lambda_{\ell} / 2} \int \frac{d E}{2 \pi}\left[B_{\ell}(E)-\bar{B}_{\ell}(E)\right], \\
& i z w_{11}(z, \lambda)-i w_{11}(t=0, \lambda)=-\sum_{\ell} e^{-i \lambda_{\ell} / 2} \int \frac{d E}{2 \pi}\left[B_{\ell}(E)-\bar{B}_{\ell}(E)\right], \\
& \left(E-E_{d}+i z-\Gamma \int \frac{d E^{\prime}}{2 \pi} \frac{1}{E-E^{\prime}+i z}\right) B_{\ell}(E) \\
& =e^{-i \lambda_{\ell} / 2} f_{\ell}(E) \Gamma_{\ell}\left(w_{00}(z, \lambda)+\sum_{\ell^{\prime}} e^{i \lambda_{\ell^{\prime}} / 2} \int \frac{d E^{\prime}}{2 \pi} \frac{\bar{B}_{\ell^{\prime}}\left(E^{\prime}\right)}{E^{\prime}-E-i z}\right) \\
& -e^{i \lambda_{\ell} / 2}\left[1-f_{\ell}(E)\right] \Gamma_{\ell}\left(w_{11}(z, \lambda)-\sum_{\ell^{\prime}} e^{-i \lambda_{\ell^{\prime}} / 2} \int \frac{d E^{\prime}}{2 \pi} \frac{\bar{B}_{\ell^{\prime}}\left(E^{\prime}\right)}{E^{\prime}-E-i z}\right) .
\end{aligned}
$$


These equations have simple analytical solutions at infinite bias, when the tunneling rates are constant or have a Lorentzian shape [19. At finite bias however, we need a numerical solution and hence must transform the function $B(E)$ into a mapping on a discrete and finite set. Two numerical solution procedures (A and B) have been independently developed.

- A Discrete energies: In our first approach [17] we choose a set of equidistant energies and approximate the integrals with sums over these energies. This requires a cutoff at some energy and is therefore most efficient for scenarios where a sufficiently small energy interval determines the transport properties.

- B Cutoff in residues: One can show that in the crucial complex half plane the quantity $B(E)$ has the same poles as the Fermi function [30]. By this knowledge the integrals can be approximated with the residues that are closest to the real axis. This leads to an inaccuracy in the value of the Fermi functions. Thus, this method is most efficient when the transport happens at energies where the left and the right Fermi function differ sufficiently.

The two methods also differed in the way the noise was evaluated:

- A Numerical time evolution: We evaluate the time evolution of the cumulant generating function $S(\lambda, t)=-\ln \left[\sum_{b} w_{b b}(\lambda, t)\right]$. After a short time, $S(\lambda, t)$ becomes linear in time and its slope $s(\lambda)$ can be determined. The current and noise can then be calculated from $\frac{d s}{d \lambda}$ and $\frac{d^{2} s}{d \lambda^{2}}$ respectively.

- B Long time expansion in Laplace space: By comparing the factors in front of the system states $w_{a b}$ in Eq. (38) we can extract a non-Markovian master equation. As soon as this is known, we can use a Taylor expansion around $z=0$ and the techniques described in Refs. 31, 32, to calculate the noise.

In approach $\mathrm{A}$ the EOM is solved in time space by performing the Markov approximation for the density matrix elements containing two dot-lead excitations. In Laplace space this corresponds to replacing all $z$ to the right of the equality sign in Eq. (A.2) with positive infinitesimals while keeping the other $z$ in the EOM. Using somewhat hand-waving arguments it is easy to realize that doing the Markov approximation at a higher-order is less of an approximation. The $z$ to the right of the equality sign in Eq. (A.2) contribute when the rest of the denominator is small. This region can be defined as a volume in the $N$-dimensional energy space, $N$ being the order at which the Markov approximation is done. As $N$ increases this volume becomes smaller compared to the entire integration volume, which reduces the effect of neglecting the $z$.

The accuracy of the $2 \mathrm{vN}$ approach and the effect of the partial Markovian approximation is investigated in Fig. 10 where the noise, i.e. the second cumulant $\left\langle\left\langle I^{2}\right\rangle\right\rangle$, is shown as a function of the bias, $V_{\text {bias }}$, for two different coupling strengths. The dot level is positioned in the middle of the bias window and we assume equal couplings to left and right lead, $\Gamma_{L}=\Gamma_{R}$. Partial Markovian results from approach A are compared with the non-Markovian results of approach B, and the exact noninteracting results of the transmission formula. For both coupling strengths it can be seen that the effect of performing the partial Markov approximation as described above, is very small. Partial Markovian calculations were also performed using approach B. This gave results indistinguishable from approach A. For weak couplings such as Fig. 10 a) the $2 \mathrm{vN}$ results agree very well with the exact transmission results as higher-order terms are of less importance (note the y-axis scale). For the 
stronger couplings of Fig. 10 b) the agreement is good in the low bias limit. As the bias is increased the phase space for higher-order processes increases which causes a discrepancy between the $2 \mathrm{vN}$ results and the exact transmission formula.
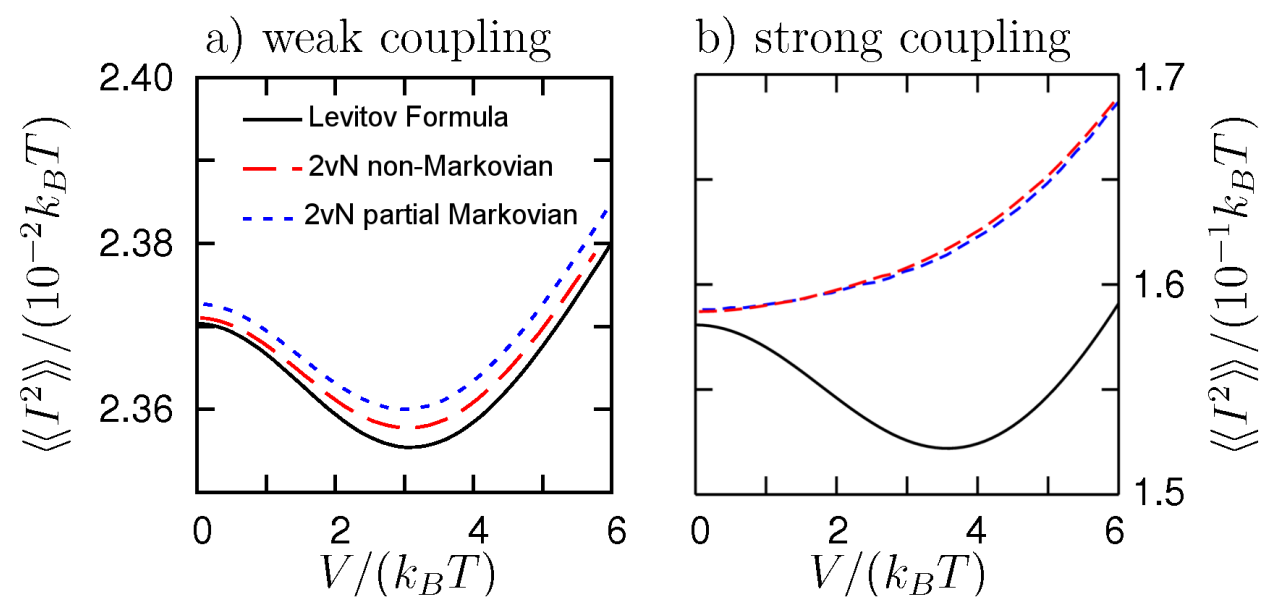

Figure 10. The noise $\left\langle\left\langle I^{2}\right\rangle\right\rangle$ for a single-level model for a) $\Gamma_{L}=\Gamma_{R}=0.1 k_{B} T$, b) $\Gamma_{L}=\Gamma_{R}=k_{B} T$, and the dot level positioned at the middle of the bias window. In the strong coupling case the neglection of higher order tunneling results in a discrepancy between the $2 \mathrm{vN}$ results and the exact transmission formula as expected.

\section{Noise and Fano factor for the canyon of current suppression}

So far the success and shortcoming of the $2 \mathrm{vN}$ method have been studied. In this section we change focus and use the partial Markovian version A of the method to calculate the noise and Fano factor of the canyon of current suppression, previously investigated experimentally [20] and theoretically [21, 33, 34, 35, 36, 37. The canyon appears as a suppression of current in both sequential and co-tunneling regimes, close to degeneracy in two-level spinless systems.

In the single-particle eigenbasis of the dot, the system Hamiltonian is given by:

$$
\begin{aligned}
\hat{H} & =\hat{H}_{\text {dot }}+\hat{H}_{\text {leads }}+\hat{H}_{T}, \\
\hat{H}_{\text {dot }} & =E_{1} d_{1}^{\dagger} d_{1}+E_{2} d_{2}^{\dagger} d_{2}+U d_{1}^{\dagger} d_{1} d_{2}^{\dagger} d_{2}, \\
\hat{H}_{\text {leads }} & =\sum_{k, \ell=L / R} E_{k} c_{k \ell}^{\dagger} c_{k \ell}, \\
\hat{H}_{T} & =\sum_{k, \ell=L / R}\left(t_{\ell 1} d_{1}^{\dagger}+t_{\ell 2} d_{2}^{\dagger}\right) c_{k \ell}+\text { H.c. },
\end{aligned}
$$

where we have assumed that the couplings $t_{\ell i}$ are independent of $k$ and $\Gamma_{\ell i}(E)=$ $2 \pi t_{\ell i}^{2} \rho_{0}$ with a constant density of states $\rho_{0}(E)=\sum_{k} \delta\left(E_{k}-E\right)$, for $-D<E_{k}<D$. In the simulations a large bandwidth $D$ is used, assuming wide conduction bands of the leads. The operators $d_{i}\left(d_{i}^{\dagger}\right)$ and $c_{k l}\left(c_{k l}^{\dagger}\right)$ are annihilation (creation) operators of electrons in the dot and leads, respectively. In Eq. (40) the charging energy $U$ is due to Coulomb repulsion between the electrons when both dot states are filled. 
Noise calculations for this system have previously been performed in Ref. 38. under the assumption that one level was very weakly coupled to the reservoirs. Here we report results for the two level system without this assumption, demonstrating the versatility of the $2 \mathrm{vN}$ approach. Noise calculations have also been performed for similar systems such as serial double quantum dots [39], albeit in the weak coupling regime.

We parametrize the energy levels as

$$
E_{1 / 2}= \pm \frac{\Delta E}{2}-E_{g}-U / 2
$$

where $\Delta E$ is the splitting between the two levels and $E_{g}$ is a common shift of the levels.

We study couplings of the type

$$
t_{L 1}=t, \quad t_{R 1}=t, \quad t_{L 2}=-a t, t_{R 2}=a t,
$$

where the asymmetry parameter $a$ is chosen to be real, as such couplings were shown to be essential for observation of the canyon [21.

Figures [11a)-c) show normalized current $\langle\langle I\rangle\rangle / V_{\text {bias }}$, normalized noise $\left\langle\left\langle I^{2}\right\rangle\right\rangle / V_{\text {bias }}$, and the Fano factor $F=\left\langle\left\langle I^{2}\right\rangle\right\rangle /\langle\langle I\rangle\rangle$ as a function of $\Delta E$ and $E_{g}$ using the parameters $V_{\text {bias }}=15 k_{B} T, \Gamma_{L 1}=\Gamma_{R 1}=k_{B} T, \Gamma_{L 2}=\Gamma_{R 2}=k_{B} T / 4$, and $U=25 k_{B} T$ corresponding to the parameters of Fig. 2 (e) in Ref. [21].

Fig. 11 a) shows the canyon of current suppression discussed in Ref. 21. To understand the next order cumulant of charge transport we look at the results for the Fano factor. The most prominent feature in the plot are the two hills located at $\Delta E / k_{B} T=-10, E_{g} / k_{B} T=5$ and $\Delta E / k_{B} T=10, E_{g} / k_{B} T=-5$. These hills result form the trapping of the electron in a low conducting state. The level configuration at $\Delta E / k_{B} T=10, E_{g} / k_{B} T=-5$, marked by a white cross in Fig. 11] c), is shown in Fig. 11]d). Here, state $|2\rangle$ of the dot is at most times filled, which corresponds to a very low conductance, as current is carried by co-tunneling processes through either the weakly coupled state $|2\rangle$ or through state $|1\rangle$ which is far from the bias window due to Coulomb interaction. However, sometimes inelastic co-tunneling events empty $|2\rangle$ and fill $|1\rangle$, resulting in a highly conductive system. As a result the electron transport is bunched which corresponds to as large noise signal and a high Fano factor. The simultaneous treatment of sequential tunneling and co-tunneling in the presence of Coulomb interaction is therefore essential for describing the charge transport in this regime. Thus, the $2 \mathrm{vN}$ approach is ideal for this type of calculations. Neglecting the effects of finite temperature and level broadening this phenomenon occurs at level configurations

$$
\begin{aligned}
& -\frac{V_{\text {bias }}}{2}<E_{1}<\frac{V_{\text {bias }}}{2}, \\
& \frac{V_{\text {bias }}}{2}<E_{1}+U, \\
& E_{2}<-\frac{V_{\text {bias }}}{2},
\end{aligned}
$$

and similar for the hill located at $\Delta E / k_{B} T=-10, E_{g} / k_{B} T=5$. For the parameters considered in Fig. 11 the limits are set by $-\frac{V_{\text {bias }}}{2}<E_{1}$ and $E_{2}<-\frac{V_{\text {bias }}}{2}$, corresponding to the areas restricted by the red lines in Fig. 11 c). Furthermore, the level splitting must be smaller than the bias to enable conservation of energy. This requirement does not impose any restrictions in Fig. 11]c) as $|\Delta E|<V_{\text {bias }}$ in this plot. For $|\Delta E|>V_{\text {bias }}$ 

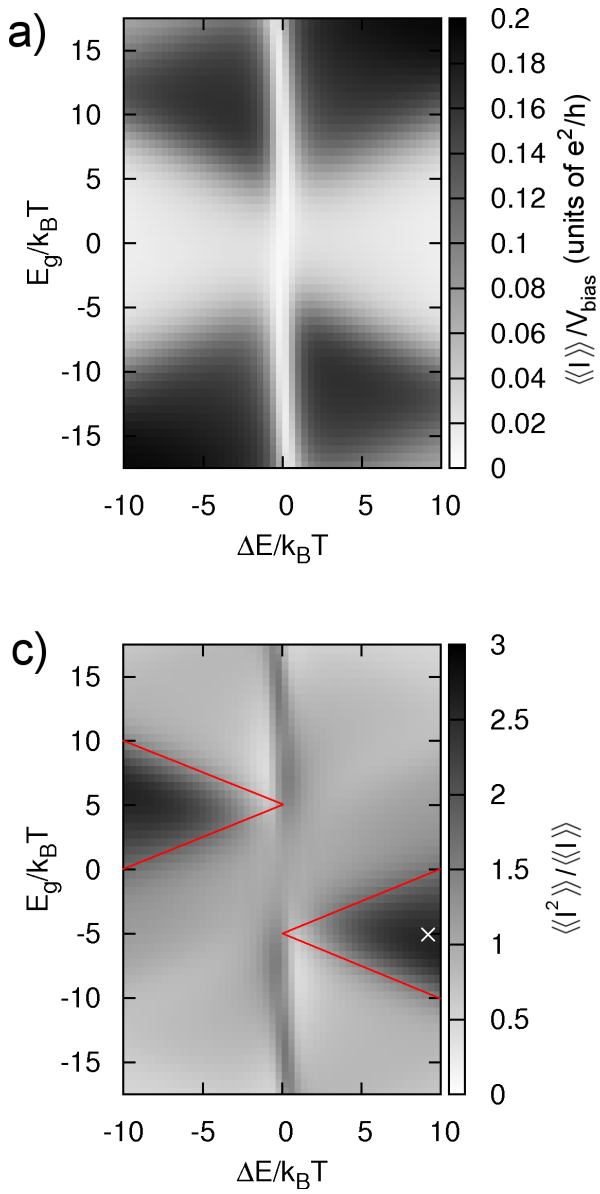

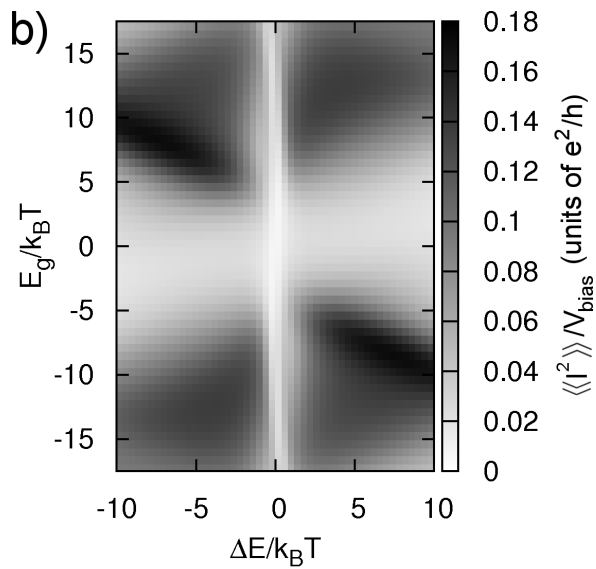

d)
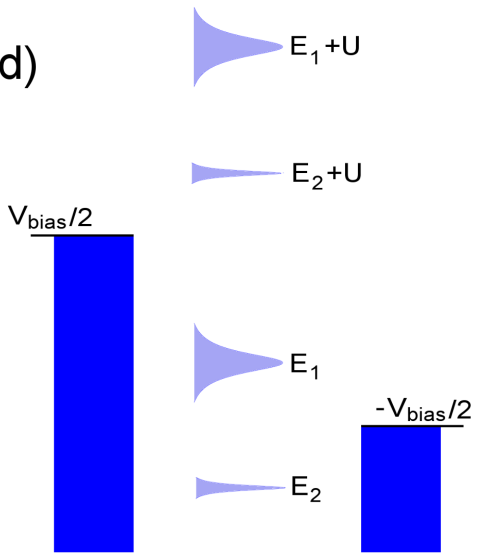

Figure 11. a) The normalized current $\langle\langle I\rangle\rangle / V_{\text {bias }}$, b) the normalized noise $\left\langle\left\langle I^{2}\right\rangle\right\rangle / V_{\text {bias }}$, in units of $e^{2} / h$, and c) the Fano factor $F=\left\langle\left\langle I^{2}\right\rangle\right\rangle /\langle\langle I\rangle\rangle$. The parameters are given by $V_{\mathrm{bias}}=15 k_{B} T, \Gamma_{L 1}=\Gamma_{R 1}=k_{B} T, \Gamma_{L 2}=\Gamma_{R 2}=$ $k_{B} T / 4$, and $U=25 k_{B} T$. d) The level positions at the white cross in Fig. c).

the current drastically decreases, and the Fano factor drops to a value close to unity as the inelastic co-tunneling processes no longer are possible, see Fig. 12 .

This phenomenon is different from the dynamical channel blockade described in Refs. 40, 41, where both levels are located within the bias window and tunneling can be described within the sequential regime. The regime considered in the current work gives a stronger bunching of electrons as the dot at most times is in a state where there is no state inside the bias window that can carry the electron. Indeed, in the regime where both states are in the bias window corresponding to the dynamical channel blockade, a Fano factor close to one is observed for the studied parameters. There is an exception to this is close to degeneracy, $\Delta E=0$. Here the Fano factor exhibits a "ridge" resulting from interference between the two dot states. When the dot states are degenerate, electrons can tunnel into any superposition of these states. Especially it can happen that an electron tunnels into a linear combination with zero coupling to the right lead. When this happens the electron is trapped in the dot 42 as it cannot 


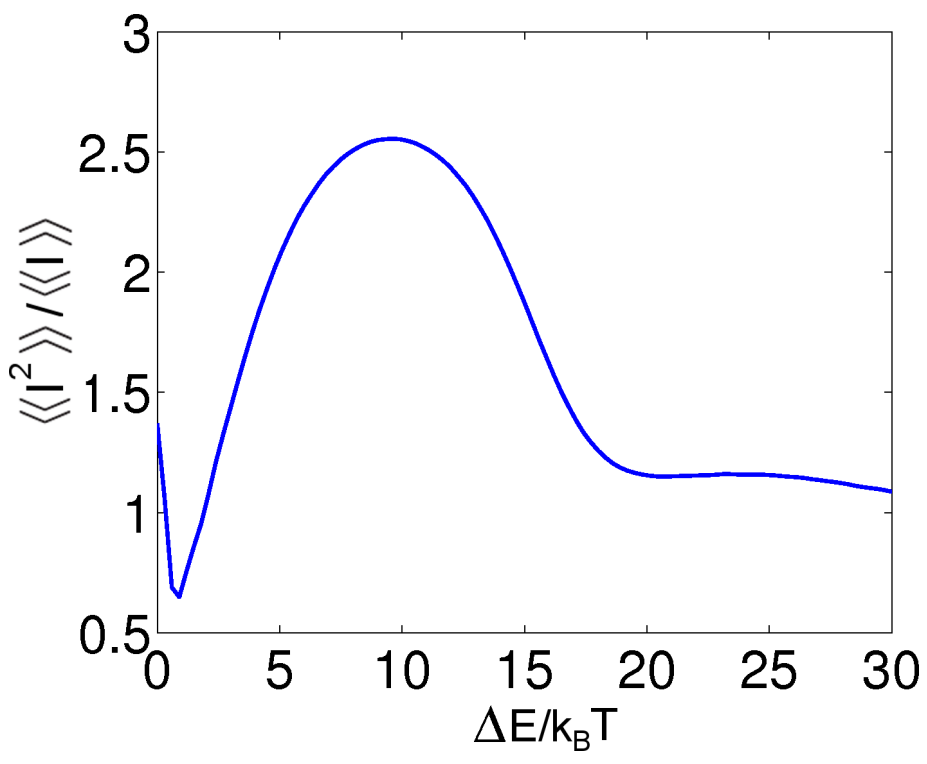

Figure 12. The Fano factor as a function of $\Delta E$ for $E_{g}=-5 k_{B} T$. The other parameters are given by $V_{\text {bias }}=15 k_{B} T, \Gamma_{L 1}=\Gamma_{R 1}=k_{B} T, \Gamma_{L 2}=\Gamma_{R 2}=$ $k_{B} T / 4$, and $U=25 k_{B} T$. For $\Delta E>V_{\text {bias }}$ inelastic co-tunneling processes are no longer possible, resulting in a lower Fano factor.

easily tunnel back to the left lead due to the high bias. This explain the canyon of current suppression in the regime where both levels are well inside the bias window. Furthermore it results in strong bunching of charge transport [43, i.e. the ridge in the Fano factor, as rare co-tunneling events allow the trapped electron to escape back to the left lead. Unlike dynamical channel blockade, this is not a modulation of the charge transport where the weakly coupled state controls the transport through the strongly coupled state. Instead it is an interference effect between the two levels.

For $-5 k_{B} T<E_{g}<5 k_{B} T$ and $\Delta E=0$, there is no state in the bias window. Even if the dot leaves the non-conducting state, its conductance is still low as current can only be carried by co-tunneling processes. Thus, the ridge is weaker in this regime.

Estimating the error in these simulations is somewhat more difficult as it is not possible to benchmark against the exact transmission formula for interacting systems. However, the results presented in Ref. [30. show that at couplings of $\Gamma=k_{B} T$ and $V_{\text {bias }}=15 k_{B} T$ the noise agrees very well with the transmission formula for the singlelevel dot. This suggests that we are in a regime where higher order tunneling events are of less importance and the $2 \mathrm{vN}$ method can be trusted to provide accurate results.

\section{Conclusions}

The $2 \mathrm{vN}$ method and the resonant tunneling approximation within the real-time diagrammatic approach were compared using Liouvillian perturbation theory. This established the equivalence between the two methods and the recently developed method of Ref. [18. Using diagrammatic techniques it was shown how diagrams of third and higher order in the lead-dot coupling energy, not included in the $2 \mathrm{vN}$ 
approach, canceled in the EOM for the single-particle reduced density matrix of noninteracting systems. This enables the exact calculation of the current. However, it was seen that to correctly reproduce the EOM for the many-body density matrix, or the Full Counting Statistics, diagrams of higher-order were needed. The discrepancy in the noise between the $2 \mathrm{vN}$ method and the exact transmission formula was investigated in the non-interacting limit. For stronger lead-dot couplings an increased discrepancy was observed at larger bias, due to an increased phase space for higher-order tunneling. Finally, the noise of the canyon of current suppression was calculated using the $2 \mathrm{vN}$ method. While the current and noise showed a canyon around level degeneracy, the Fano factor exhibited a ridge, as well as local maxima due to electron bunching.

\section{Acknowledgments}

This work was supported by the Swedish research council and Deutsche Forschungsgesellschaft via SFB 910 and project BR 1528/8. We are grateful to Martin Leijnse and Tomáš Novotný for useful discussions and comments on the manuscript.

\section{Appendix A. Equation of motion for the 2vN approach including counting fields}

Using the notation of Sec. 2 the EOM, originally derived in Ref. [19, is given by

$$
\begin{aligned}
\left(z-E_{b}+E_{b^{\prime}}\right) w_{b b^{\prime}}= & +\sum_{a, k \ell} e^{\mathrm{i} \lambda_{\ell} / 2}\left(T_{b a}(k \ell) \chi_{a b^{\prime}}(k \ell)-\phi_{b a}(k \ell) T_{b^{\prime} a}^{*}(k \ell)\right) \\
& +\sum_{c, k \ell} e^{-\mathrm{i} \lambda_{\ell} / 2}\left(T_{c b}^{*}(k \ell) \phi_{c b^{\prime}}(k \ell)-\chi_{b c}(k \ell) T_{c b^{\prime}}(k \ell)\right)
\end{aligned}
$$




$$
\begin{aligned}
& \left(z-E_{c}+E_{b}+E_{k}\right) \phi_{c b}(k \ell)= \\
& +\sum_{b^{\prime}} e^{\mathrm{i} \lambda_{\ell} / 2} T_{c b^{\prime}}(k \ell) f_{k \ell} w_{b^{\prime} b}-\sum_{c^{\prime}} f_{k \ell}^{*} w_{c c^{\prime}} T_{c^{\prime} b}(k \ell) e^{-\mathrm{i} \lambda_{\ell} / 2} \\
& +\sum_{a, b^{\prime}, k^{\prime} \ell^{\prime}} \frac{\left[e^{\mathrm{i} \lambda_{\ell^{\prime}}} T_{c b^{\prime}}\left(k^{\prime} \ell^{\prime}\right) f_{k^{\prime} \ell^{\prime}} \phi_{b^{\prime} a}(k \ell)-f_{k \ell}^{*} \phi_{c b^{\prime}}\left(k^{\prime} \ell^{\prime}\right) T_{b^{\prime} a}(k \ell) e^{\mathrm{i}\left(\lambda_{\ell^{\prime}}-\lambda_{\ell}\right) / 2}\right] T_{b a}^{*}\left(k^{\prime} \ell^{\prime}\right)}{E_{k}+E_{k^{\prime}}-\left(E_{c}-E_{a}\right)+z} \\
& +\sum_{a, b^{\prime}, k^{\prime} \ell^{\prime}} \frac{\left[f_{k^{\prime} \ell^{\prime}}^{*} \phi_{c b^{\prime}}(k \ell) T_{b^{\prime} a}\left(k^{\prime} \ell^{\prime}\right)-e^{\mathrm{i}\left(\lambda_{\ell^{\prime}}+\lambda_{\ell}\right) / 2} T_{c b^{\prime}}(k \ell) f_{k \ell} \phi_{b^{\prime} a}\left(k^{\prime} \ell^{\prime}\right)\right] T_{b a}^{*}\left(k^{\prime} \ell^{\prime}\right)}{E_{k}+E_{k^{\prime}}-\left(E_{c}-E_{a}\right)+z} \\
& +\sum_{a, b^{\prime}, k^{\prime} \ell^{\prime}} \frac{T_{c b^{\prime}}\left(k^{\prime} \ell^{\prime}\right)\left[f_{k^{\prime} \ell^{\prime}} \phi_{b^{\prime} a}(k \ell) T_{b a}^{*}\left(k^{\prime} \ell^{\prime}\right) e^{\mathrm{i} \lambda_{\ell^{\prime}}}-e^{\mathrm{i}\left(\lambda_{\ell^{\prime}}+\lambda_{\ell}\right) / 2} T_{b^{\prime} a}(k \ell) f_{k \ell} \chi_{a b}\left(k^{\prime} \ell^{\prime}\right)\right]}{E_{k}-E_{k^{\prime}}-\left(E_{b^{\prime}}-E_{b}\right)+z} \\
& +\sum_{b^{\prime}, c^{\prime}, k^{\prime} \ell^{\prime}} \frac{T_{c b^{\prime}}\left(k^{\prime} \ell^{\prime}\right)\left[T_{c^{\prime} b^{\prime}}^{*}\left(k^{\prime} \ell^{\prime}\right) f_{k^{\prime} \ell^{\prime}}^{*} \phi_{c^{\prime} b}(k \ell)-f_{k \ell}^{*} \chi_{b^{\prime} c^{\prime}}\left(k^{\prime} \ell^{\prime}\right) T_{c^{\prime} b}(k \ell) e^{\mathrm{i}\left(\lambda_{\ell^{\prime}}-\lambda_{\ell}\right) / 2}\right]}{E_{k}-E_{k^{\prime}}-\left(E_{b^{\prime}}-E_{b}\right)+z} \\
& +\sum_{b^{\prime}, c^{\prime}, k^{\prime} \ell^{\prime}} \frac{\left[f_{k^{\prime} \ell^{\prime}} \phi_{c b^{\prime}}(k \ell) T_{c^{\prime} b^{\prime}}^{*}\left(k^{\prime} \ell^{\prime}\right)-e^{\mathrm{i}\left(\lambda_{\ell}-\lambda_{\ell^{\prime}}\right) / 2} T_{c b^{\prime}}(k \ell) f_{k \ell} \chi_{b^{\prime} c^{\prime}}\left(k^{\prime} \ell^{\prime}\right)\right] T_{c^{\prime} b}\left(k^{\prime} \ell^{\prime}\right)}{E_{k}-E_{k^{\prime}}-\left(E_{c}-E_{c^{\prime}}\right)+z} \\
& +\sum_{c^{\prime}, d, k^{\prime} \ell^{\prime}} \frac{\left[e^{-\mathrm{i} \lambda_{\ell^{\prime}}} T_{d c}^{*}\left(k^{\prime} \ell^{\prime}\right) f_{k^{\prime} \ell^{\prime}}^{*} \phi_{d c^{\prime}}(k \ell)-f_{k \ell}^{*} \chi_{c d}\left(k^{\prime} \ell^{\prime}\right) T_{d c^{\prime}}(k \ell) e^{-\mathrm{i}\left(\lambda_{\ell}+\lambda_{\ell}^{\prime}\right) / 2}\right] T_{c^{\prime} b}\left(k^{\prime} \ell^{\prime}\right)}{E_{k}-E_{k^{\prime}}-\left(E_{c}-E_{c^{\prime}}\right)+z} \\
& +\sum_{c^{\prime}, d, k^{\prime} \ell^{\prime}} \frac{T_{d c}^{*}\left(k^{\prime} \ell^{\prime}\right)\left[f_{k^{\prime} \ell^{\prime}}^{*} \phi_{d c^{\prime}}(k \ell) T_{c^{\prime} b}\left(k^{\prime} \ell^{\prime}\right) e^{-\mathrm{i} \lambda_{\ell^{\prime}}}-e^{\mathrm{i}\left(\lambda_{\ell}-\lambda_{\ell^{\prime}}\right) / 2} T_{d c^{\prime}}(k \ell) f_{k \ell} \phi_{c^{\prime} b}\left(k^{\prime} \ell^{\prime}\right)\right]}{E_{k}+E_{k^{\prime}}-\left(E_{d}-E_{b}\right)+z} \\
& +\sum_{c^{\prime}, d, k^{\prime} \ell^{\prime}} \frac{T_{d c}^{*}\left(k^{\prime} \ell^{\prime}\right)\left[T_{d c^{\prime}}\left(k^{\prime} \ell^{\prime}\right) f_{k^{\prime} \ell^{\prime}} \phi_{c^{\prime} b}(k \ell)-f_{k \ell}^{*} \phi_{d c^{\prime}}\left(k^{\prime} \ell^{\prime}\right) T_{c^{\prime} b}(k \ell) e^{-\mathrm{i}\left(\lambda_{\ell^{\prime}}+\lambda_{\ell}\right) / 2}\right]}{E_{k}+E_{k^{\prime}}-\left(E_{d}-E_{b}\right)+z},
\end{aligned}
$$

here $f_{k \ell}^{*}=1-f_{k \ell}$. The EOM for $\chi$ can be obtained by taking the complex conjugate of Eq. A.2 followed by replacing $\lambda_{\ell}$ with $-\lambda_{\ell}, \phi_{b a}^{*}$ with $\chi_{a b}$, and $\chi_{a b}^{*}$ with $\phi_{b a}$. It should be noted that $\phi, \chi$, and $w$ all depend on the counting fields $\lambda_{\ell}$.

\section{Appendix B. LPT current blocks}

The blocks used in the current and noise expressions, Eqs. (30) and (31), can be derived from the following two primitive blocks:

$$
\begin{aligned}
& \mathcal{J}^{(1)}\left(z_{1}, z_{0}\right)=\overleftrightarrow{G-X^{\prime}}+\stackrel{X^{\prime}-G}{-}
\end{aligned}
$$

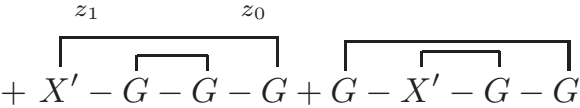

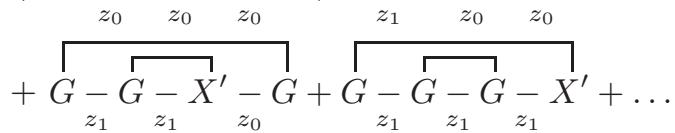




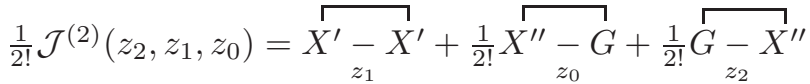

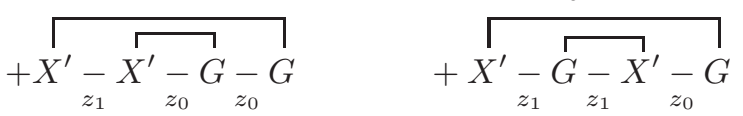

$$
\begin{aligned}
& +\overbrace{}^{\prime}-\stackrel{z^{\prime}-G}{z_{z_{1}}}-X^{\prime}
\end{aligned}
$$

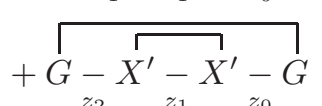

$$
\begin{aligned}
& +\overbrace{z_{2}-\overbrace{z_{2}}^{z_{2}}-X_{z_{1}}^{\prime}}^{z_{1}^{\prime}} \\
& +\frac{1}{2 !} \stackrel{\overbrace{}}{G-X_{z_{2}}^{\prime \prime}-G}-G \\
& +\frac{1}{2 !} \stackrel{\overbrace{z_{2}} \underset{z_{2}}{G-G}-X_{z_{2}}^{\prime \prime}}{z^{\prime \prime}}+\ldots,
\end{aligned}
$$

where we have written out explicitly only the sequential and direct co-tunneling contributions. In the diagrams, $X^{\prime}$ and $X^{\prime \prime}$ represent the first and second derivatives of the $\lambda$-dependent tunnel vertex evaluated at $\lambda=0$. The blocks of Eq. (30) and Eq. (31) are obtained as

$$
\begin{aligned}
& J_{1 b}(\omega)=\mathcal{J}^{(1)}(-i \omega, 0) \\
& J_{1 a}(\omega)=\mathcal{J}^{(1)}(-i \omega,-i \omega) \\
& J_{2 a}(\omega)=\frac{1}{2} \mathcal{J}^{(2)}(-i \omega,-i \omega, 0)
\end{aligned}
$$

where in the latter two blocks, we can throw away all diagrams with a leftmost $G$ or $X^{\prime \prime}$-vertex due to the occurrence of these blocks to the far left in the current and noise expressions 25 .

\section{References}

[1] Blanter Y M and Büttiker M 2000 Physics Reports 3361

[2] Levitov L S, Lee H and Lesovik G B 1996 J. Math. Phys. 374845

[3] Tu M W Y and Zhang W-M 2008 Phys. Rev. B 78235311

[4] Jin J S, Tu M T W, Zhang W-M and Yan Y J 2010 New Journal of Physics 12083013

[5] Jin J S, Zhang W M, Li X Q and Yan Y J 2012 Noise spectrum of quantum transport through quantum dots: a combined effect of non-markovian and cotunneling processes Preprint arXiv:1105.0136v2

[6] König J, Schoeller H and Schön G 1997 Phys. Rev. Lett. 784482

[7] König J, Schoeller H and Schön G 1998 Phys. Rev. B 587882

[8] Wangsness R K and Bloch F 1953 Phys. Rev. 89728

[9] Bloch F 1957 Phys. Rev. 1051206

[10] Redfield A G 1965 Adv. Magn. Reson. 11

[11] Timm C 2008 Phys. Rev. B 77195416

[12] Koller S, Grifoni M, Leijnse M and Wegewijs M R 2010 Phys. Rev. B 82235307

[13] Averin D V and Odintsov A A 1989 Phys. Lett. A 140251

[14] Geerligs L J, Averin D V and Mooij J E 1990 Phys. Rev. Lett. 653037

[15] Pedersen J N, Lassen B, Wacker A and Hettler M H 2007 Phys. Rev. B 75235314

[16] Hansen T, Mujica V and Ratner M A 2008 Nano Letters 83525

[17] Pedersen J N and Wacker A 2005 Phys. Rev. B 72 195330; Pedersen J N and Wacker 2010 Physica E 42595

[18] Jin J S, Zheng X and Yan Y 2008 J. Chem. Phys. 128234703

[19] Zedler P, Emary C, Brandes T and Novotný T 2011 Phys. Rev. B 84233303

[20] Nilsson H A, Karlström O, Larsson M, Caroff P, Pedersen J N, Samuelson L, Wacker A, Wernersson L-E and Xu H Q 2010 Phys. Rev. Lett. 104186804 
[21] Karlström O, Pedersen J N, Samuelsson P and Wacker A 2011 Phys. Rev. B 83205412

[22] Gogolin A O and Komnik A 2006 Phys. Rev. B 73195301

[23] Leijnse M and Wegewijs M R Phys. Rev. B 78235424

[24] Schoeller H 2009 Eur. Phys. J. Spec. Top 168179

[25] Emary C 2011 Journal of Physics: Condensed Matter 23025304

[26] König J, Schmid J, Schoeller H and Schön G 1996 Phys. Rev. B 5416820

[27] Emary C 2009 Phys. Rev. B 80235306

[28] Emary C and Aguado R 2011 Phys. Rev. B 84085425

[29] Braun M, König J and Martinek J 2006 Phys. Rev. B 74075328

[30] Zedler P 2011 Master equations in transport statistics PhD thesis, Technische Universität Berlin

[31] Flindt C, Novotný T, Braggio A, Sassetti M and Jauho A-P 2008 Phys. Rev. Lett. 100150601

[32] Flindt C, Novotný T, Braggio A and Jauho A-P 2010 Phys. Rev. B 82155407

[33] Kashcheyevs V, Schiller A, Aharony A and Entin-Wohlman O 2007 Phys. Rev. B 75115313

[34] Meden V and Marquardt F 2006 Phys. Rev. Lett. 96146801

[35] Lee H-W and Kim S 2007 Phys. Rev. Lett. 98186805

[36] Silva A, Oreg Y and Gefen Y 2002 Phys. Rev. B 66195316

[37] Silvestrov P G and Imry Y 2007 Phys. Rev. B 75115335

[38] Carmi A and Oreg Y 2012 Phys. Rev. B 85045325

[39] Kießlich G, Schöll E, Brandes T, Hohls F and Haug R J 2007 Phys. Rev. Lett 99206602

[40] Cottet A, Belzig W and Bruder C Phys. Rev. B 70115315

[41] Urban D and König J 2009 Phys. Rev. B 79165319

[42] Li F, Li X-Q, Zhang W-M and Gurvitz S A 2009 Europhys. Lett. 8837001

[43] Li F, Jiao H, Luo J, Li X-Q and Gurvitz S A 2009 Physica E 411707 